\title{
A Neuromuscular Model of Human Locomotion Combines Spinal Reflex Circuits with Voluntary Movements
}

\author{
Rachid Ramadan, Hartmut Geyer, John J. Jeka, Gregor Schöner, Hendrik Reimann
}

September 26, 2021

\begin{abstract}
Existing models of human walking use low-level reflexes or neural oscillators to generate movement. While appropriate to generate the stable, rhythmic movement patterns of steady-state walking, these models lack the ability to change their movement patterns or spontaneously generate new movements in the specific, goal-directed way characteristic of voluntary movements. Here we present a neuromuscular model of human locomotion that bridges this gap and combines the ability to execute goal directed movements with the generation of stable, rhythmic movement patterns that are required for robust locomotion. The model represents goals for voluntary movements of the swing leg on the task level of swing leg joint kinematics. Smooth movements plans towards the goal configuration are generated on the task level and transformed into descending motor commands that execute the planned movements, using internal models. The movement goals and plans are updated in real time based on sensory feedback and task constraints. On the spinal level, the descending commands during the swing phase are integrated with a generic stretch reflex for each muscle. Stance leg control solely relies on dedicated spinal reflex pathways. Spinal reflexes stimulate Hill-type muscles that actuate a biomechanical model with eight internal joints and six free-body degrees of freedom. The model is able to generate voluntary, goaldirected reaching movements with the swing leg and combine multiple movements in a rhythmic sequence. During walking, the swing leg is moved in a goal-directed manner to a target that is updated in real-time based on sensory feedback to maintain upright balance, while the stance leg is stabilized by low-level reflexes and a behavioral organization switching between swing and stance control for each leg. With this combination of reflexive stance leg and voluntary, goal-directed control of the swing leg, the model controller generates rhythmic, stable walking patterns in which the swing leg movement can be flexibly updated in real-time to step over or around obstacles.
\end{abstract}

\section{Introduction}

Walking is one of the most common movements humans perform every day. Walking consists of putting one foot in front of the other while moving the body forward. Most of the time walking does not require attention. But when walking in complex terrain, we are able to precisely step to suitable locations. When someone bumps into us, we are able to modify our normal movement pattern to maintain upright balance. In these situations, we are able to quickly and smoothly transition to conscious control of the usually largely reflexive walking movement. The motor control of walking as a movement that is usually habitual and reflexive, sometimes voluntary and goal-directed, and often somewhere in-between is currently not well understood. In this paper, we present a neuromechanical model for generating walking movements that is capable of covering the whole range of walking movements between these two poles.

\subsection{Human Walking as a Voluntary Movement}

Human movement shows amazing flexibility. We can perform a wide variety of tasks that require different movement patterns and coordination between body parts. Meaningful tasks usually require us to move a body part or tool to a goal position, such as the finger to a button or a screwdriver to a screw. Many tasks also contain additional requirements for timing or force, e.g. catching a ball in the air or hitting a nail with a hammer. The human nervous system routinely solves complex movements tasks in situations that it never 
specifically encountered before, using sensory information to generate a movement plan and update it during execution.

Humans are able to flexibly modify the basic pattern of their gait cycle during walking (Steele et al., 2012; Ackermann and van den Bogert, 2012). At a high level, a walking movement pattern can be quantified by variables like speed and heading direction, and the length, width, duration and frequency of steps, typically referred to as gait parameters (Levine et al., 2012). Humans can generally choose these parameters as desired. They can change direction, walk fast or slow, with narrow or wide steps and a slow or fast pace, etc. (Inman et al., 1981). In addition to this high-level flexibility of gait patterns, humans are also able to choose how exactly they perform each low-level limb movement. Stepping to a fixed location in a fixed time can be performed with a variety of trajectories for the swing foot. We can swing the foot higher to step over an obstacle, or closer to the stance leg to step around an object. We can also choose to walk with bent knees, with the foot rotated in or out, or tip-toe by limiting ground contact to the balls of the foot and keep the heels up.

\subsection{Stability and Upright Balance}

One aspect of moving a body part to a target is the ability to confine movement to only the desired body part, while keeping the rest of the body stable and un-moving. Pushing a button requires not only muscles along the arm and shoulder to move the finger to the button, but also muscles along the trunk and legs to stabilize the rest of the body, so that the contact force at the finger results in moving the button in rather than the body away (Woollacott et al., 1984). Muscle activation measurements reveal that when initiating such a manipulation movement while standing, muscles along the legs and trunk that stabilize the body activate earlier than muscles along the shoulder and arms that move the arm (Aruin et al., 1998). Stability is an integral part of the motor system that is integrated into the movement plan at all stages (Bouisset and Zattara, 1987).

Stability is especially important for the upright body as a whole. When the body is upright during standing or walking, failure to stabilize it properly can lead to a fall, resulting in impact with the ground and serious injury. Yet for walking, "not moving" is not an option. We cannot keep parts of the body static relative to the environment, because locomotion of the whole body to a different place is the functional goal. The task for the nervous system is to generate a stable movement pattern for the whole body, transporting it with a relatively constant velocity from one point to another, while keeping movements in other directions to a minimum. To solve the main task of locomotion, the legs need to generate forces against the ground, initially to accelerate the body in the direction of travel and reach a steady state of motion, then to regulate the body movement around the steady state movement pattern and correct deviations from it. To prevent falls, the legs need to generate vertical forces that keep the body mass at a certain height, and also horizontal forces that regulate the body movement in the direction orthogonal to the direction of travel. Both of these requirements need to be combined into a cyclical pattern of moving one leg ahead in a step while supporting the body weight with the other one, then shifting weight and the role of the legs.

\subsection{Habitual Control}

Despite the flexibility to choose from a large range of walking patterns and movements, normal human walking is usually highly repetitive, with few variations. Humans will generally choose a walking pattern and then adhere to it for longer stretches of time, with gait parameters relatively stable on a time scale of minutes (Dean, 1965). One factor driving this long-term stability of walking patterns is energy efficiency. The "cost of transport" of using metabolic energy to move from one place to another depends on the walking speed, with large cost at high and low speeds, and lower cost at medium speeds (Ralston, 1958). Humans usually choose to walk near the speed where this metabolic cost of transport is minimal (Ralston, 1958; Browning et al., 2006; Summerside et al., 2018). A second factor affecting the choice of gait pattern is balance (Bauby and Kuo, 2000; Reimann et al., 2018a). Walking with increased step width increases the base of support during double stance, so the body is passively more stable (Donelan et al., 2004). But higher step width also leads to larger average displacement between the body center of mass and the stance foot during single stance, increasing the lever arm of the gravitational force pulling the body down, and thus the muscle forces required to counter gravity and keep us upright. Higher muscle forces require more 
metabolic energy, so there is a trade-off between balance and metabolic cost, where gait patterns that are more stable are also less efficient Donelan et al. (2001). Balance is also actively maintained by changing the foot placement relative to the average gait pattern based on the current state of the body in space (Wang and Srinivasan, 2014; Bruijn and van Dieën, 2018; Reimann et al., 2018b). This active control of foot placement aggregates high-level sensory information about the body in space from the visual and vestibular and proprioceptive systems (Peterka, 2002) and maps it to changes in foot placement. This mode of control is neither reflexive in the narrow sense nor voluntary or conscious, but similar to online updating to a new target during a reaching movement (Scott, 2004).

The choice of gait pattern is different across different groups of people. Older people tend to walk more slowly (Osoba et al., 2019; Reimann et al., 2020; Pijnappels et al., 2008). People with Parkinson's Disease tend to take short, shuffling steps (Jankovic, 2008). People with Cerebral Palsy often swing their legs out to the side much more than typical (Sutherland and Davids, 1993). While there are reasonable explanations for some of these gait pattern changes, the underlying causes are often not well understood. One reason for this limited understanding is the complexity of the problem. Walking is a biomechanically complex motor pattern with many moving parts (Nielsen, 2003). The concrete choice of motor pattern depends on many different factors, including metabolic energy cost, avoiding muscle fatigue, stability and control of upright balance, and external constraints such as obstacles and the condition of the walking surface (Kirtley et al., 1985; Prentice et al., 2004; Voloshina et al., 2013; Hunter et al., 2010; Matthis and Fajen, 2014; Summerside et al., 2018; Kung et al., 2018). While motor control of walking is largely sub-conscious, cognitive processes also play a role, and secondary tasks during walking have routinely been shown to affect gait parameters and balance control (Matthis and Fajen, 2014).

\subsection{Modeling Walking Control}

To understand the interactions between different factors that drive the choice of walking movement pattern, we need a computational model that includes all factors of interest (Allen and Ting, 2016; De Groote and Falisse, 2021). Such a model allows us to manipulate individual factors and observe the resulting changes in the walking pattern directly in simulation studies (Reimann et al., 2020). Existing neuromechanical models of walking largely focus on the generation of rhythmic movement patterns and balance control. The rhythmic movement patterns are either generated by neural oscillators (Taga, 1995a; Van der Noot et al., 2018) or by a finite state machine switching between different movement states depending on ground contact (Günther and Ruder, 2003; Geyer and Herr, 2010). These existing models have some degree of flexibility. Some models can walk at different speeds (Taga, 1995b; Song and Geyer, 2015; Van der Noot et al., 2018), change direction (Van der Noot et al., 2018), and step over obstacles (Taga, 1998; Song and Geyer, 2015). This can be achieved by re-parameterizing a model, essentially optimizing a large number of neuromechanical parameters to walk at a range of different speeds, and then switching between these parameter sets, or interpolating between them, to change speed during walking (Song and Geyer, 2015; Van der Noot et al., 2018; Di Russo et al., 2021). Another approach is to modulate the central neural drive of a model to oscillate faster (Taga, 1995b; Van der Noot et al., 2018). Similar techniques can be used to step over obstacles, either increasing the gain between the central oscillator and the flexor muscles of the swing leg hip and knee (Taga, 1998), or the target flexion angle for a reflex at the same joints, with similar effect (Song and Geyer, 2015). These approaches generally provide solutions for one specific problem, e.g. walking at different speeds or stepping over an obstacle, but do not generalize directly to related problems, such as walking at different cadences or stepping around an obstacle, rather than over it. Humans, in contrast, are not only capable of flexibly modulating gait parameters or the path of the swing foot, but can spontaneously walk in novel patterns, which they never used or observed before.

Our goal is to develop a neuromechanical model of walking that shows a similar degree of flexibility as humans, in that it can generate any desired walking pattern. We postulate that the key limitation of current walking models is that they are almost completely spinal, and lack cortical motor planning and control. These high-level features are usually studied as part of upper extremity reaching movements (Kalaska et al., 1997; Sabes, 2000; d'Avella and Lacquaniti, 2013). Some researchers have pointed out the duality of steps as (i) part of a cyclical movement pattern of the whole body for locomotion and (ii) a reaching movement with the foot (Reynolds and Day, 2005b,a; Smid and den Otter, 2013; Mowbray et al., 2019; Barton et al., 2019). Experimental evidence indicates that stepping movements during walking are generated rhythmically using 
low-level, reflexive structures (Mutha, 2017; Ivanenko et al., 2006; Zehr and Stein, 1999; Stein, 1991). On the other hand, these movements can be precisely and efficiently modulated by high-level influences when desired, e.g. to step to a specific target or around an obstacle (Zhang et al., 2020).

Here we present a model extension that attempts to bridge this gap between existing neuromechanical models of walking and the ability to plan and execute voluntary movements with the leg. The key innovation in our model is an explicit movement plan for the swing leg on task level. The high-level movement plan is executed by transforming the planned movements into descending commands that integrate with the low-level, reflexive control architecture of the spinal cord, using internal models to account for dynamic interaction forces and properties of the muscles and spinal reflexes. For the stance leg, we use an existing solution of dedicated spinal reflex modules that generate the appropriate muscle activation with minimal high-level input (Song and Geyer, 2015). We show that this model is able to generate voluntary swing leg movements, and to integrate these swing leg movements into a rhythmic walking pattern, modulated by high-level feedback to maintain upright balance.

\section{Methods}

The model spans multiple levels, across high-level movement planning and coordination, spinal reflex arcs, muscle physiology and skeletal biomechanics. Figure 1 provides an overview. A finite state machine organizes the model and switches between swing and stance phase control for each leg. In the supraspinal layer, a volition module represents task-level movement goals, a planning module generates motor plans to reach the goal state and a balance module updates the movement plan based on real-time sensory feedback about the body in space. An internal model then transforms the high-level motor plan into descending motor commands that interface with the spinal cord to execute the planned movement. In the spinal cord, the swing leg is controlled by a generic stretch reflex that is modulated by the descending commands, while the stance leg control is purely reflexive. Reflexes stimulate Hill-type muscles that actuate a biomechanical model.

The key innovation here is the integration of the volition module in the supraspinal layer that prescribes movement goals with the other components. While the volition module itself is relatively simple, the main challenge in the development of this model was to integrate the task-level movement goals with the low-level spinal reflex control modules so that the resulting system can combine stable, repetitive walking movements with voluntary, goal-directed movements that solve tasks represented in the volition module. The finite state machine, balance control, spinal reflexes, muscle model and biomechanics are all modeled with standard solutions from textbooks or the literature. Each module is described in detail below.

\subsection{High-level Control}

\subsubsection{Behavioral organization}

Walking requires the sequential execution of different movements for each limb, organized in a cyclical pattern (Moissenet et al., 2019; Fukuchi et al., 2018). We organize the model behavior in three phases per leg, (1) early swing, (2) late swing and (3) stance. A finite state machine generates transitions between these phases based on sensory information of ground contact and internal timing. The early swing phase is initiated by the detection of ground contact of the contralateral leg $(3 \rightarrow 1)$. Transition to the late swing phase occurs after a fixed time of $0.3 \mathrm{~s}(1 \rightarrow 2)$. The late swing phase lasts until ground contact is detected, leading to a transition to the stance phase $(2 \rightarrow 3)$. This system is functionally equivalent to one with four global states of early swing and late swing for each leg, used, e.g. by Yin et al. (2007), since ground contact detection of the swing leg triggers transitions. During swing, the leg is controlled in a goal-directed way based on a movement plan (see Section 2.1.4 below). During stance, the leg is controlled in a purely reflexive way (see Section 2.2.2 below). 
bioRxiv preprint doi: https://doi.org/10.1101/2021.09.26.461864; this version posted September 26, 2021. The copyright holder for this preprint (which was not certified by peer review) is the author/funder, who has granted bioRxiv a license to display the preprint in perpetuity. It is made available under aCC-BY-NC-ND 4.0 International license.

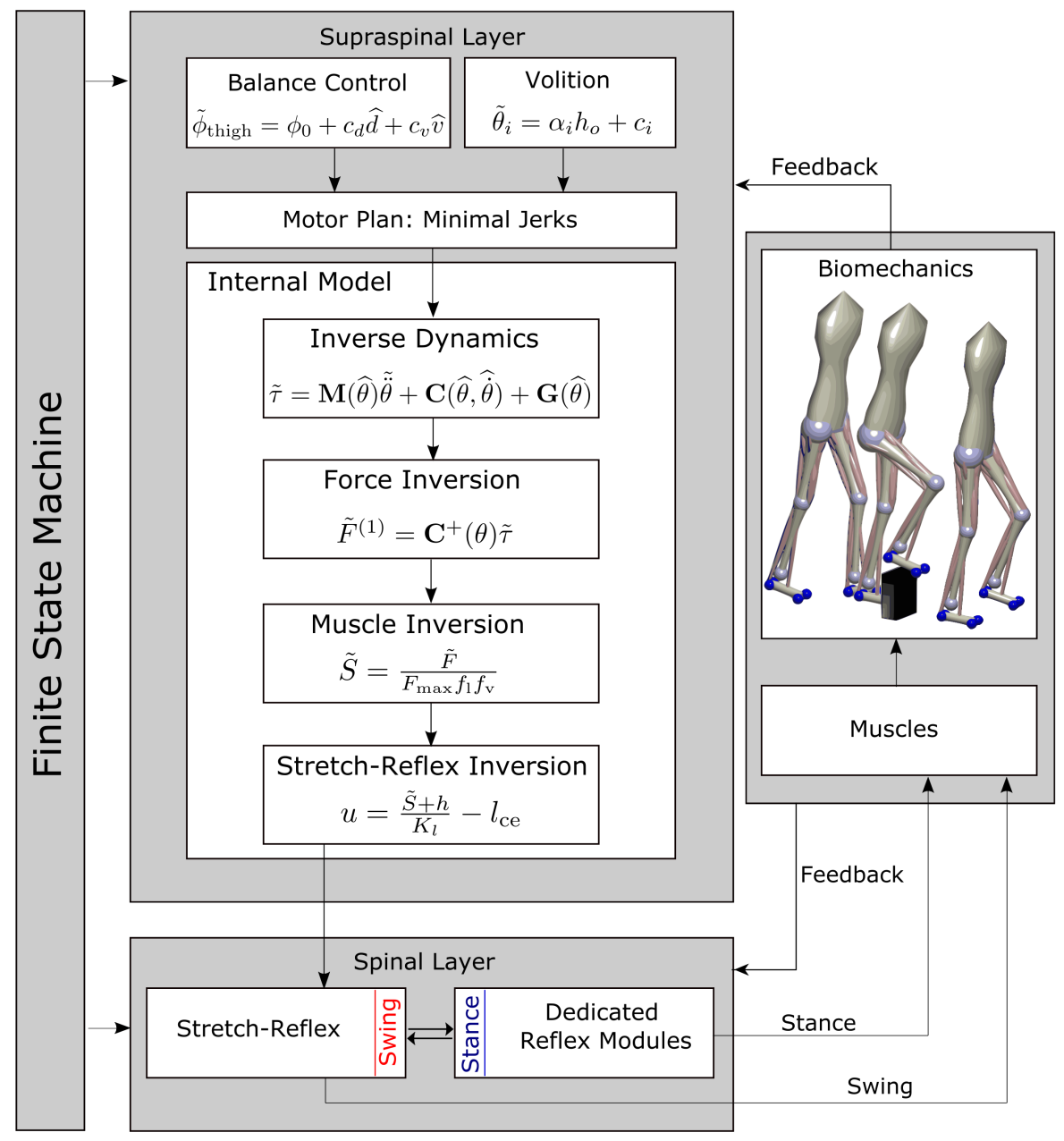

Figure 1: Overview of the model architecture. In the supraspinal layer, a balance control equation defines target joint angles for the swing leg at mid-swing and heel-strike. The target joint angles can be modified to perform volitional, goal-directed movements. A movement plan towards these target joint configurations is generated by minimal jerk trajectories that can be updated during execution. An internal inverse model comprising biomechanics, muscle moment arms, muscle activation properties and the spinal stretch reflex produces descending commands that realize the planned movement. The descending commands are integrated with the stretch reflex in the spinal layer. Stance leg control is realized with five dedicated reflex modules Song and Geyer (2015). Reflex outputs are the applied to the biomechanical model that provides feedback to the controller. A finite state machine organizes switches between early swing phase, late swing phase and stance phase. 


\subsubsection{Volition}

A goal for a voluntary movement of the swing limb is a desired configuration of the limb kinematics, represented by a vector of desired joint angles $\tilde{\theta}_{i} \cdot{ }^{1}$ In principle, this goal configuration can be anything, and we will probe the generation of movements to randomly chosen configurations (see Section 3.1). For walking, the goal configuration for each movement phase must be appropriate to generate a stable gait pattern, and we use evolutionary optimization to find suitable configurations. During individual steps, the goal configurations can be modified to address specific tasks, such as obstacle avoidance (see Section 3.2 or balance control (see Section 2.1.3).

\subsubsection{Balance Control}

Maintaining balance requires the integration of state feedback about the body in space into the movement plan. We use position and velocity feedback of the trunk center to update the desired target orientation of the thigh in space. Following Yin et al. (2007), we use the control law

$$
\tilde{\phi}_{\text {thigh }}=\phi_{0}+c_{d} \widehat{d}+c_{v} \widehat{v}
$$

where $\phi_{\text {thigh }}$ is the desired orientation of the swing leg thigh, $\phi_{0}$ is a constant offset, $\widehat{d}=d(t-\Delta t)$ are the time-delayed horizontal displacement from the center of pressure $(\mathrm{CoP})$ to the trunk segment center, $\widehat{v}=v(t-\Delta t)$ the time-delayed rate of change of that displacement and $c_{d}$ and $c_{v}$ are feedback gains. Equation 1 is applied independently for the sagittal and frontal plane orientation of the thigh. We then calculate target joint angles for each DoFs of the hip joint

$$
\tilde{\theta}_{\text {hip }}=\tilde{\phi}_{\text {thigh }}-\phi_{\text {trunk }}
$$

by subtracting the trunk orientation $\theta_{\text {trunk, world }}$ from desired thigh orientation, again separately in the frontal and sagittal planes. Note that if the target joint angle for the knee in the late swing phase is close to zero, the thigh angle will correspond closely to the swing leg angle in space, which is relevant for balance.

\subsubsection{Movement Planning}

The swing leg is controlled in a goal-directed way according to a task-level motor plan. The task-level goal is a kinematic configuration of the swing leg, defined by the swing leg joint angles, combined with a target time at which the goal configuration should be reached. Goal configurations and target times are different for early and late swing phase and can be updated to maintain whole-body balance (see Section 2.1.3 above) or to generate specific voluntary movements. The leg will typically be far away from the goal configuration at the onset of each movement phase, and there is an infinite number of possible movement trajectories that will fulfill the task constraints. Human movements are generally smooth and avoid unnecessary spikes in force and acceleration, and a standard way to plan such movements are minimum jerk trajectories (Hogan, 1984).

For a given combination of initial state

$$
X_{0}=\left(\theta_{0}, \dot{\theta}_{0}, \ddot{\theta}_{0}\right)
$$

and goal state

$$
X_{\text {tgt }}=(\tilde{\theta}, \tilde{\dot{\theta}}, \tilde{\ddot{\theta}})
$$

of joint angles, velocities and accelerations for a single joint angle $\theta$, and a movement duration $T$, the minimum jerk trajectory is a 5 th-order polynomial

$$
x(t)=\sum_{k=0}^{5} a_{k} t^{k}
$$

\footnotetext{
${ }^{1}$ The tilde in $\tilde{\theta}_{i}$ indicates that this is a desired state of the joint angle, in contrast to the actual joint angle $\theta_{i}$. We will use this convention of the tilde to denote desired states throughout the rest of the text.
} 
with parameters $a_{k}$ that fulfill the constraints

$$
(x(0), \dot{x}(0), \ddot{x}(0))=X_{0}, \quad(x(T), \dot{x}(T), \ddot{x}(T))=X_{\text {tgt }},
$$

which can be computed analytically depending on $T, X_{0}$ and $X_{\text {tgt }}$. We use a version of the minimal jerk approach that allows changes in target states and time before the movement is complete. For every moment in time $t$, we regard the current state estimate

$$
X_{\mathrm{t}}=(\widehat{\theta}(t), \widehat{\dot{\theta}}(t), \widehat{\ddot{\theta}}(t))=(\theta(t), \dot{\theta}(t), \ddot{\theta}(t))
$$

as the initial state of a new movement and compute parameters $a_{k}(t)$ such that in the remaining time $(T-t)$, the movement reaches the target state $X_{\text {tgt }}$. From the resulting parameters $a_{k}(t)$, we compute the jerks

$$
j(t)=\dddot{x}_{\mathrm{t}}\left(t^{\prime}\right)=\frac{d^{3} x_{t}\left(t^{\prime}\right)}{d t^{\prime 3}} .
$$

For very small remaining movement times $T-t<0.03 \mathrm{~s}$, we stop updating the motor plan. Integrating these jerks over time yields a desired joint acceleration

$$
\tilde{\ddot{\theta}}_{\text {swing }}(t)=a(t)=\int j\left(t^{\prime}\right) d t^{\prime}
$$

to be realized with descending motor commands. The tilde indicates that this is a planned, or desired, joint acceleration, in contrast to the actually realized joint acceleration that will be a combination of active and passive muscle-tendon forces, gravity, ground reaction forces and interaction torques. We will keep this convention to use a tilde to indicate planned or desired values for a variable from here on. By applying this procedure of updating the planned trajectory based on the estimated state during the entire movement, we are able to adapt the initial minimal jerk trajectory to account for any external or internal perturbation and correct the resulting errors.

We use this procedure to generate a minimum jerk trajectory for each degree of freedom in the swing leg that moves the leg to the target configuration in the given time. The target joint angles for early swing and late swing are part of the parameters set that is determined by evolutionary optimization (see Section 2.4 below).

\subsubsection{Transformation into Descending Motor Commands}

The motor plan is represented by a minimum-jerk trajectory that moves the joint configuration to the desired state in the remaining time (see Section 2.1.4 above). At each point in time, this planned trajectory defines a

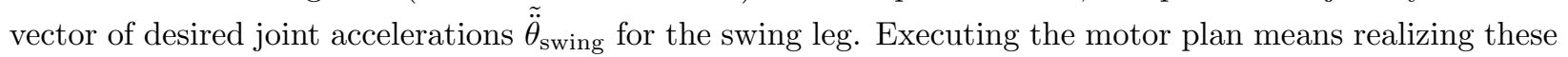
planned joint accelerations. Here we describe how this vector of desired joint accelerations is transformed into a descending motor command that executes the motor plan. We solve this problem using inverse models of the biomechanics, muscle force dynamics and spinal reflex arcs, with simplifying assumptions.

Inverse Dynamics. The biomechanical Equation of Motion (17) relates joint accelerations to joint torques. We augment the planned vector of joint accelerations for the four degrees of freedom in the swing leg by zeros (Siciliano and Khatib, 2008) in the components for the stance leg and the six free-body degrees of freedom of the trunk to get a vector of joint acceleration for the full 14-DoF model

$$
\tilde{\ddot{\theta}}_{\text {full }}=\left[\begin{array}{c}
0_{6 \times 1} \\
\tilde{\ddot{\theta}}_{\text {swing }} \\
0_{4 \times 1}
\end{array}\right]
$$

and use an Equation (17) to get a planned joint torque vector

$$
\tilde{\tau}_{\text {full }}=\mathbf{M}(\widehat{\theta}) \tilde{\ddot{\theta}}_{\text {full }}+\mathbf{C}(\widehat{\theta}, \widehat{\dot{\theta}})+\mathbf{G}(\widehat{\theta}),
$$


where $\widehat{\theta}(t)=\theta\left(t+\delta_{\theta}\right)$ and $\widehat{\dot{\theta}}(t)=\dot{\theta}\left(t+\delta_{\dot{\theta}}\right)$ are time-delayed sensor estimates of the body configuration and rate of change. We then take the swing leg components $\tilde{\tau}_{\text {swing }}$ of

$$
\tilde{\tau}_{\text {full }}=\left[\begin{array}{c}
\tilde{\tau}_{\text {trunk }} \\
\tilde{\tau}_{\text {swing }} \\
\tilde{\tau}_{\text {stance }}
\end{array}\right]
$$

as the desired joint torques for the swing leg that will execute the motor plan. We implement Equation 11 using the Inverse Dynamics block in Simulink.

Muscle Moment Arm Inversion. In order to obtain a set of muscle forces $\tilde{F}$ that generate the desired joint torques $\tilde{\tau}$, we use the Moore-Penrose pseudo-inverse (Murray et al., 1994) of the moment arm matrix $C$ to get

$$
\tilde{F}^{(1)}=\mathbf{C}^{+}(\theta) \tilde{\tau} .
$$

The resulting force vector $\tilde{F}^{(1)}$, however, can contain negative forces, which cannot physically be generated by muscles. Instead of using a computationally intensive solution like the non-negative least squares (Lawson and Hanson, 1995), we use an iterative approximation. We separate the negative part of the resulting forces $\tilde{F}_{-}^{(1)}$, consisting of the muscle forces with negative signs from the positive part of the forces $\tilde{F}_{+}^{(1)}$ and compute the joint torques produced only by the negative forces $\tau_{-}^{(1)}=\mathbf{C}(\theta) F_{-}^{(1)}$. We then apply Equation 13 again on these torques, getting $\tilde{F}^{(2)}=-\mathbf{C}^{+}(\theta) \tau_{-}^{(1)}$, which will also contain both positive and negative forces. Iterating this procedure leads to progressively smaller remaining negative forces $\tilde{F}_{-}^{(i)}$. We apply this procedure for a total number of 7 iterations and sum up all positive forces to obtain $\tilde{F}=\sum_{i=1}^{7} \tilde{F}_{+}^{(i)}$ as a force vector that will approximately generate the joint torques $\tilde{\tau}$.

Inverse Muscle Model. The force generated by a muscle depends on its activation level and its current length and velocity. We compute the activation needed to generate the desired muscle force $\tilde{F}$ by inverting the muscle model, with some simplifications. We neglect the low pass filtering of the muscle activation which models the excitation-contraction coupling, setting $\tilde{S}=\tilde{A}$. We approximate the total muscle force $F_{s e}$ with the force of the contractile element $F_{\text {se }}$, neglecting the contributions of the passive buffer and parallel elements. This is reasonable because the buffer and the parallel element are active only when muscles are extensively stretched or compressed, which is usually not the case during walking.

We then invert Equation 19 to calculate the neural stimulation $\tilde{S}$ needed to generate the desired muscle force as

$$
\tilde{S}=\frac{\tilde{F}}{F_{\max } f_{1} f_{\mathrm{v}}} .
$$

All terms here are 22-dimensional vectors, with one component per muscle, and the operations are executed element-wise.

Spinal Stretch Reflex Modulation. The descending commands from the high-level motor areas have to interface with the reflex arcs in the spinal cord to generate muscle activation levels that will execute the planned movement. Described in detail in Section 2.2 below, we assume that the descending command both (i) directly creates muscle activation leading to contraction and (ii) shifts the reference point of the spinal stretch reflex to a new location corresponding to the contracted state. We solve Equation 16, which models this behavior, to calculate a descending motor command

$$
u=\frac{\tilde{S}+h}{K_{l}}-l_{\text {ce }}
$$

Note that while we neglected the velocity term in the stretch reflex $K_{v}\left(\widehat{v}_{\mathrm{ce}}+\dot{u}\right)$ here, it is this velocitydependent term that will initially create the direct muscle activation, determined by $\dot{u}$. This descending motor command $u$ will interact with the spinal stretch reflex to generate the desired muscle activation $\tilde{S}$ that executes the motor plan. 


\subsection{Spinal Control}

Spinal control consists of reflexive neural feedback loops, i.e. feedback laws that generate neural activation proportional to low-level proprioceptive signals about muscle length, velocity or force, modulated by descending commands on a slower time-scale. We treat control of the leg during swing separately from the control of the leg during stance. While the swing leg is controlled by a combination of descending commands and a generic stretch reflex, the stance leg is controlled by specialized reflex modules that implement a specific function.

\subsubsection{Swing Leg}

During swing, the neural stimulation for each muscle is generated by a generic stretch reflex

$$
S=\left[K_{l}\left(\widehat{l}_{\mathrm{ce}}+u\right)+K_{v}\left(\widehat{v}_{\mathrm{ce}}+\dot{u}\right)-h\right]^{+},
$$

where $\widehat{l}_{\text {ce }}$ and $\widehat{v}_{\text {ce }}$ are proprioceptive signals from muscle spindles that estimate the stretch and stretch rate of change of the muscle contractile element, $K_{l}$ and $K_{v}$ are gain factors, $h$ is the resting level activation of the $\alpha$-motorneuron and $u$ and $\dot{u}$ are the descending motor command and its rate of change.

Note that the descending command $u$ acts as a threshold for the reflex loop and the rate of change $\dot{u}$ is used for relative damping. When the descending command $u$ increases to contract the muscle, both $u$ and $\dot{u}$ will increase initially, generating a stimulation burst that is mostly driven by the rate of change $\dot{u}$. While formulated as a single stretch reflex with relative damping here, this is functionally equivalent to a formulation where the $\alpha$-motorneuron activation level is determined by a sum of a spinal stretch reflex and a descending motor command, as used in other models (Feldman, 1986; Gribble et al., 1998; Günther and Ruder, 2003; Kistemaker et al., 2007; Buhrmann and Di Paolo, 2014).

This principle of modulating a generic stretch reflex with a descending motor command leads to the flexibility to execute motor plans for goal-directed movements via appropriately chosen descending commands, combined with the robustness of a stretch reflex that provides a level of postural stability to the muscle-joint system in situations where it is not part of a goal-directed movement.

\subsubsection{Stance Leg}

During stance, the leg is controlled by purely spinal mechanisms, without modulation by descending motor commands and without the flexibility to execute goal-directed movements. Proprioceptive information from different muscles and joints is mapped to proportional muscle activation in a set of dedicated neural control laws that implement specific functions, organized in five modules following Song and Geyer (2015). Briefly, the modules (1) generate compliant, spring-like leg behavior, (2) prevent knee overextension, (3) keep the trunk upright, (4) compensate interaction torques from swing leg movements and (5) dorsiflex the ankle joint to prevent hyperextension. Please refer to Song and Geyer (2015) for details.

\subsection{Muscoskeletal Mechanics}

\subsubsection{Body Model}

The body model represents a person of $180 \mathrm{~cm}$ height and $80 \mathrm{~kg}$ weight. It is composed of seven body segments, eight degrees of freedom (DoF) and 22 muscle-tendon units (MTU). Body segments comprise two thighs, shanks and feet, and a trunk segment that represents the entire upper body, including head and arms (Song and Geyer, 2015). Revolute joints link the body segments with two DoFs at each hip (pitch and roll), one DoF at the knees (pitch) and one DoF at the ankles (pitch). The equation of motion

$$
\tau=\mathbf{M}(\theta) \ddot{\theta}+\mathbf{C}(\theta, \dot{\theta})+\mathbf{G}(\theta)+\mathbf{T}_{\mathbf{e x t}}
$$

relates joint torques $\tau$, gravitational torques $\mathbf{G}$ and external torques $\mathbf{T}_{\text {ext }}$ to joint accelerations $\ddot{\theta}$, where $\mathbf{M}$ represents the mass matrix and $\mathbf{C}$ the velocity dependent terms. Joint accelerations $\ddot{\theta}$ and torques $\tau$ are 14-dimensional vectors, with the eight internal DoFs and six free-body DoFs for translation and orientation of the trunk segment. Note that the six free-body DoFs of the trunk are un-actuated. Geometry and inertia of the body segments are adopted from Song and Geyer (2015). 
Each leg is actuated by eleven Hill-type MTUs that are either mono- or biarticulary (see Section 2.3.2 below for details). Nine MTUs actuate the three pitch joints (hip, knee, ankle) and two MTUs actuate the hip roll joint. Pitch joint muscles model the lumped hip flexors, glutei, hamstrings, rectus femoris, vasti, biceps femoris short head, gastrocnemius, soleus and tibialis anterior. Roll joint muscles represent the lumped hip adductors and hip abductors. Muscles forces translate into joint torques via state-dependent moment arms that are adopted from Song and Geyer (2015), via

$$
\tau=\mathbf{C} F,
$$

where $F$ is the 22 -dimensional vector of muscle forces and $C$ is the $14 \times 22$ matrix of moment arms.

\subsubsection{Muscle-Tendon Units}

Each muscle tendon unit (MTU) is composed of a parallel element (PE), a buffer element (BE), a contractile element $(\mathrm{CE})$ and a serial elastic element (SE). We provide an overview here and refer the reader to Geyer and Herr (2010) for details. The contractile element is the actual active muscle element. It is innervated by the $\alpha$-motorneurons and exerts the force

$$
F_{\text {ce }}=A F_{\max } f_{1}\left(l_{\mathrm{ce}}\right) f_{\mathrm{v}}\left(v_{\mathrm{ce}}\right) .
$$

Here, $F_{\max }$ is the maximum isometric force, $f_{1}\left(l_{\mathrm{ce}}\right)$ and $f_{\mathrm{v}}\left(v_{\mathrm{ce}}\right)$ are the force-length and force-velocity relationships and $A$ is the muscle activation level. The serial element models the tendon and applies the generated forces $F_{s e}$ to the body. The parallel element passively prevents the muscle from being stretched extensively and exerts a force $F_{\mathrm{pe}}\left(l_{\mathrm{ce}}\right)$ after the muscle lengths exceeds a certain maximal length. In contrast, The buffer element is a passive element that prevents the muscle from being compressed too much. It generates the force $F_{\text {be }}\left(l_{\text {ce }}\right)$ only after the muscle length shortens below a certain minimal length. Muscle activation $A$ is modeled as a first-order low-pass filtered copy of the neural stimulation $S$ representing the $\alpha$-motorneuron output

$$
A=S-\tau_{A} \frac{\mathrm{d} A}{\mathrm{~d} t}
$$

where $\tau_{A}$ is a time constant Gribble et al. (1998). The total force $F_{\mathrm{mtu}}$ generated by a MTU is given by

$$
F_{\mathrm{mtu}}=F_{\mathrm{se}}=F_{\mathrm{ce}}+F_{\mathrm{pe}}-F_{\mathrm{be}} .
$$

\subsubsection{Ground Contact Forces}

Ground contacts at each foot are modeled with four contact points, two at the heel and two at the front of the foot, with a lateral displacement of $5 \mathrm{~cm}$ between the two points at the heel and $10 \mathrm{~cm}$ at the front. We compute ground reaction forces by using the inbuilt MATLAB Spatial Contact Force block. Contact parameters are chosen to simulate an asphalt surface.

\subsection{Parameters and Tuning}

The model contains a large number of parameters for different components of the model. Some of these parameters are constrained by the neurophysiological literature and set to constant values based on estimates. To determine the other parameters, we use an evolutionary optimization algorithm similar to the one used inSong and Geyer (2015), based on the covariance-matrix adaptation technique (Hansen, 2006), using the cost function

$$
J= \begin{cases}2 c_{0}-x_{\text {fall }} & \text { if fall } \\ c_{0}+d_{\text {steady }} & \text { else }\end{cases}
$$

The first part of the cost function generates basic walking without falling and the second part generates steady locomotion. The constant $c_{0}=10^{3}$ is a normalization factor and $d_{\text {steady }}$ measures the "steadyness" of the gait. We calculate $d_{\text {steady }}$ as

$$
d_{\text {steady }}=\sum_{j=n-2}^{n} \sum_{i=1}^{\operatorname{limb}}\left[p_{i}\left(\mathrm{HS}_{j}\right)-p_{i}\left(\mathrm{HS}_{j-1}\right)\right],
$$




\begin{tabular}{|c|c|}
\hline Parameter & value $(\mathrm{s})$ \\
\hline$d_{\theta}$ & 0.01 \\
$d_{\dot{\theta}}$ & 0.01 \\
$d_{\ddot{\theta}}$ & 0.01 \\
$d_{\text {muscle length }}$ & 0.01 \\
$d_{\text {muscle velocity }}$ & 0.01 \\
$d_{\text {balance control }}$ & 0.1 \\
$d_{u}$ & 0.0025 \\
$d_{\dot{u}}$ & 0.0025 \\
$d_{l}$ & 0.0025 \\
$d_{v}$ & 0.0025 \\
\hline
\end{tabular}

Table 1: Time Delays.

with $p_{i}$ being the relative Cartesian position of the $i$-th limb and $\mathrm{HS}_{j}$ being the $j$ th-last left heelstrike.

We optimize a total amount of 52 parameters. The same set of parameters is used for all experiments described in the results section.

\section{Results}

The model generates stable walking behavior with a movement speed of about $1.3 \mathrm{~m} / \mathrm{s}$. The walking pattern roughly matches human data. Figure 2 compares joint angle trajectories across one gait cycle averaged over a $100 \mathrm{~s}$ walk to human walking data from a public data set (Fukuchi et al., 2018). The human data is from $\mathrm{N}=24$ healthy young participants (10 female, age $27.6 \pm 4.4$ years, height $171.1 \pm 10.5 \mathrm{~cm}$, and mass 68.4 $\pm 12.2 \mathrm{~kg}$ ) walking overground at their self-selected, comfortable speed. Panel A shows hip flexion angle for the model (blue) with human data (orange). The overall shape of the model trajectory matches human data. At about $90 \%$ of the gait cycle, the model flexes the hip more strongly than the average experimental data. The model movements are also less smooth than the experimental data. Panel B shows the knee flexion angle. Again, the model generally follows the human pattern. During the stance phase, the model exhibits two sharp peaks while human data shows one wider peak in contrast. During swing, humans extend their leg a little earlier then the model does. Panel $\mathrm{C}$ shows the hip adduction angle. Here, the overall shape of the model data differs from human data. The model trajectory is less smooth and has less overall range of motion throughout the gait cycle. Note, however, that the hip adduction in humans is quite variable, and despite the structural differences, the model data lies within the confidence interval of human data during a large part of the gait cycle. Panel D shows the ankle flexion angle. The overall pattern of the model trajectories differs significantly from the human data. The ankle dorsiflexion peak is slightly after mid-stance, much earlier than in the human data. In swing, the model shows consistently higher dorsiflexion than humans.

In order to investigate the robustness of the models walking behavior, we exposed it to external perturbations in the form of force pulses of increasing strengths applied at the center of the trunk segment in different directions. Perturbations started at foot contact, lasted for $0.2 \mathrm{~s}$, and were directed forward, backward, medially or laterally. Force amplitude was ramped up until the model failed to maintain balance after the perturbation, starting at $50 \mathrm{~N}$ and increasing in steps of $50 \mathrm{~N}$. After the model fell, we decreased the step size to $5 \mathrm{~N}$ from the previous value, until it fell again. The maximal force the model was able to withstand without falling was $340 \mathrm{~N}$ for lateral, $305 \mathrm{~N}$ for medial, $165 \mathrm{~N}$ for forward and $130 \mathrm{~N}$ for backward pushes.

\subsection{Swing Leg Movement}

We evaluate the ability of the model to plan and execute voluntary movements with the swing leg in three simulation studies. For each movement type, we demonstrate that the control of voluntary movement works and the limb follows the movement trajectory as planned. To isolate the swing leg and remove balance control as a factor for these stimulation studies, we passively stabilized the trunk segment by fixing its position in 
bioRxiv preprint doi: https://doi.org/10.1101/2021.09.26.461864; this version posted September 26, 2021. The copyright holder for this preprint (which was not certified by peer review) is the author/funder, who has granted bioRxiv a license to display the preprint in perpetuity. It is made available under aCC-BY-NC-ND 4.0 International license.
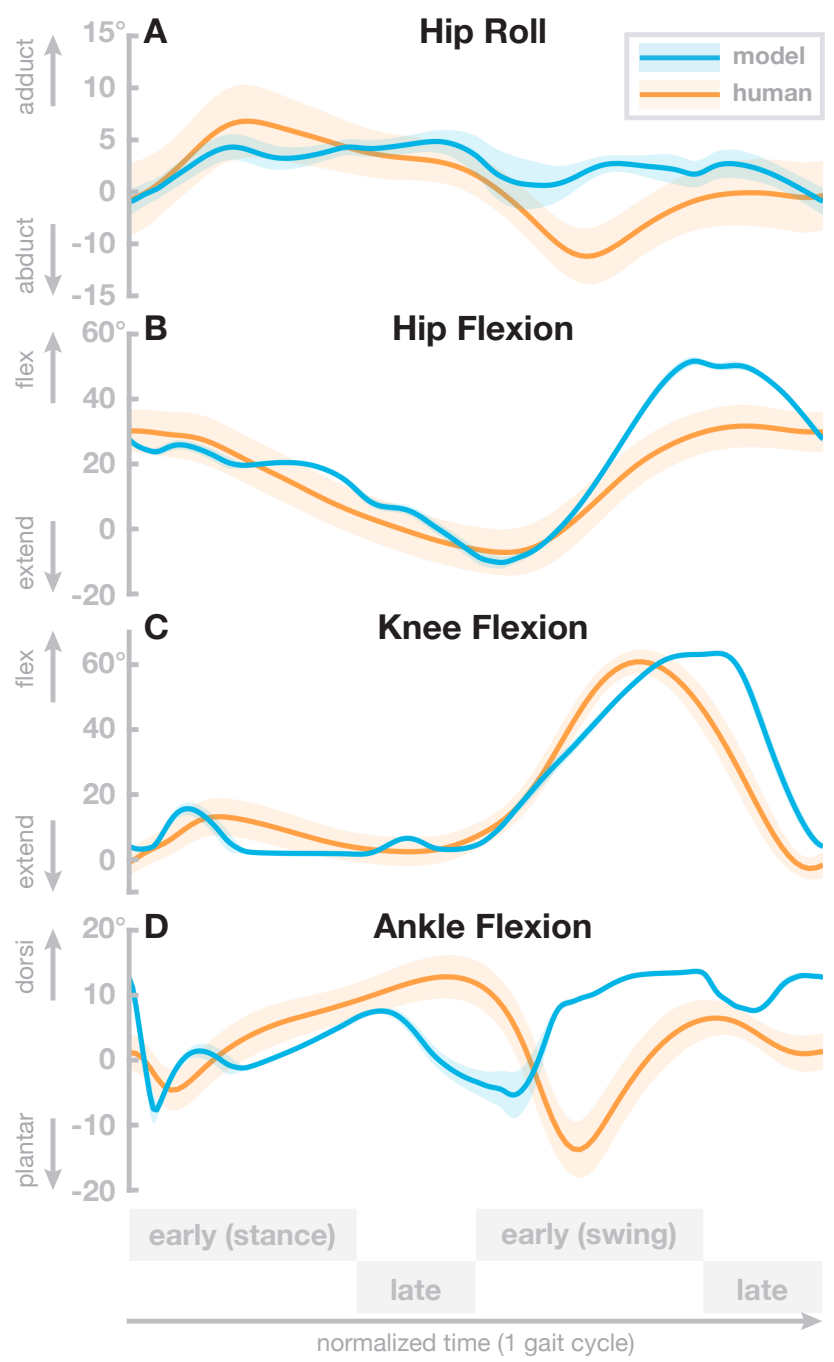

Figure 2: Comparison with human data. Averaged hip pitch, hip roll, knee and ankle joint angle trajectories for human data (orange) and model data (blue). The model data are averaged over 100 seconds of steady state walking. Human data are taken from Fukuchi et al. (2018). Solid lines are means and shaded areas are 95\% confidence intervals, across participants for the human data and across gait cycles for the model. 


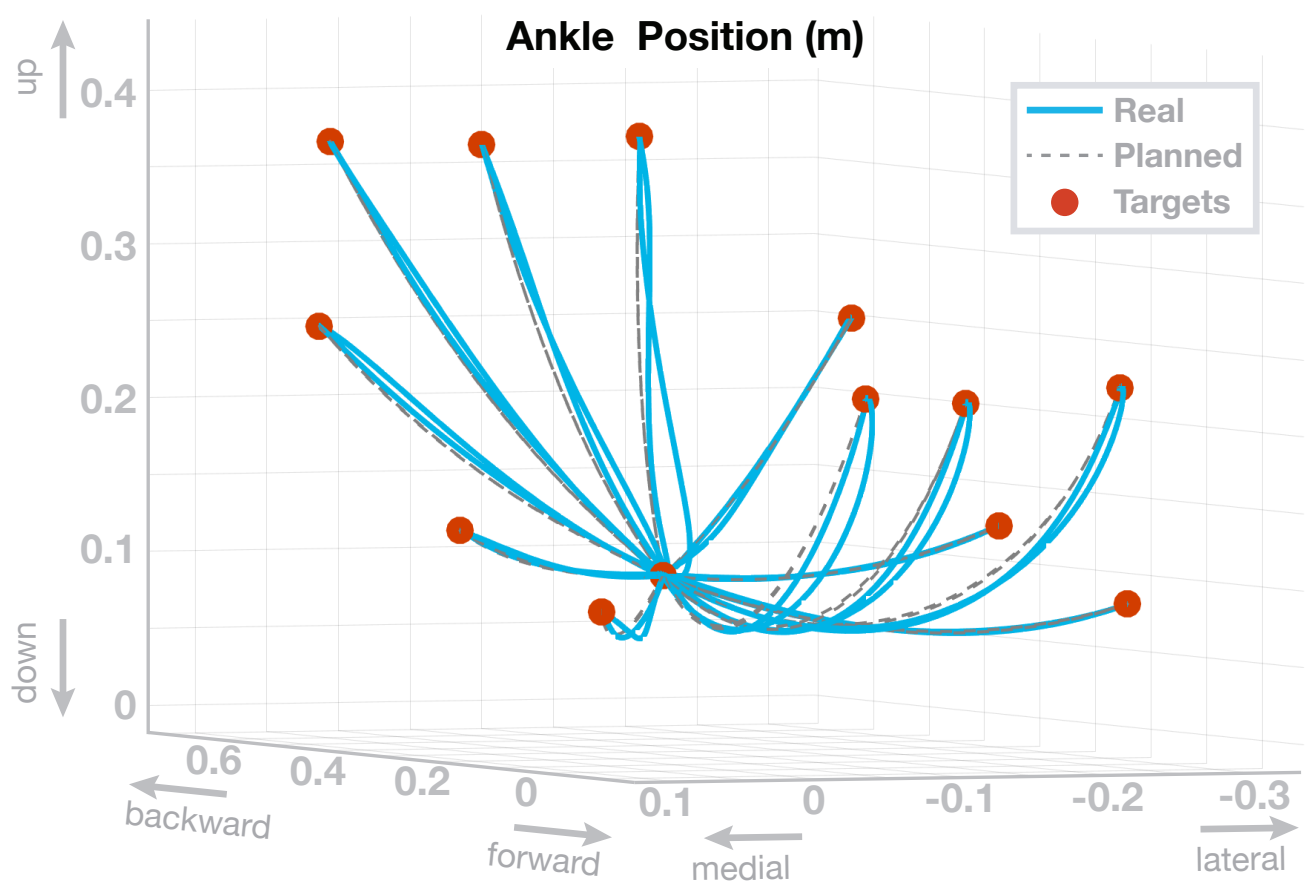

Figure 3: Swing leg ankle paths for a sequence of twelve center-out-return movements with passively stabilized trunk. The dashed lines show the planned paths and the blue lines show the realized ankle paths.

space.

In the first simulation study, we show that the model can perform individual reaching movements with the foot. The model performs a sequence of center-out reaching movements with the foot to twelve different target locations, followed by a return movement to the center location. Target locations were defined as positions for the ankle and transformed into joint space using the inverse kinematics solution in the MATLAB RigidBodyTree toolbox. The movement plan in joint space from the current to the target configuration was then generated as described in Section 2.1.4 above. The specific target locations were chosen to cover a large portion of the workspace, without being too close to the limits, resulting in path lengths between roughly $0.20-0.55 \mathrm{~m}$. Each single movement segment had a duration of $0.5 \mathrm{~s}$. Figure 3 shows the resulting movement paths of the ankle position in workspace for this sequence of reaching movements. The ankle always reaches the target positions reasonably well. The largest deviation from the planned path is at the start of the first movement, to the top right target, which is due to the muscles being initialized without tension. The paths match the reference paths with an overall root-mean-squared error of $0.003 \mathrm{~m}$ between the planned and the actual ankle position.

In the second simulation study,

the model performs repetitive goal-directed movements between two points in joint space over $10 \mathrm{~s}$, following sinusoid profiles with $1 \mathrm{~Hz}$ for each joint. Figure 4 shows the resulting joint angle trajectories (solid lines) and the planned trajectories for each joint (dashed lines). The real joint angle trajectories are a good fit of the planned movement, with only the ankle joint showing more than minimal deviation of the real trajectories from the movement plan.

In a third simulation study, we explore the flexibility of the model to generate goal-directed reaching movements with the foot between randomly chosen points in the joint space at a wide range of different speeds. For each movement, the target configuration was drawn from a uniform distribution over the interval from $15-85 \%$ of the joint range of motion for each joint. This margin was chosen relatively large, to prevent extreme body configurations. For ten different movement times ranging from $0.1-0.6 \mathrm{~s}$, we simulated 100 randomized movements each. We quantified performance as the root mean squared error between the actual and the planned trajectory. Figure 5 shows the average error for the different movement speeds in joint space. The error is high for very fast movements. For normal movement times of 0.25 seconds and above, 


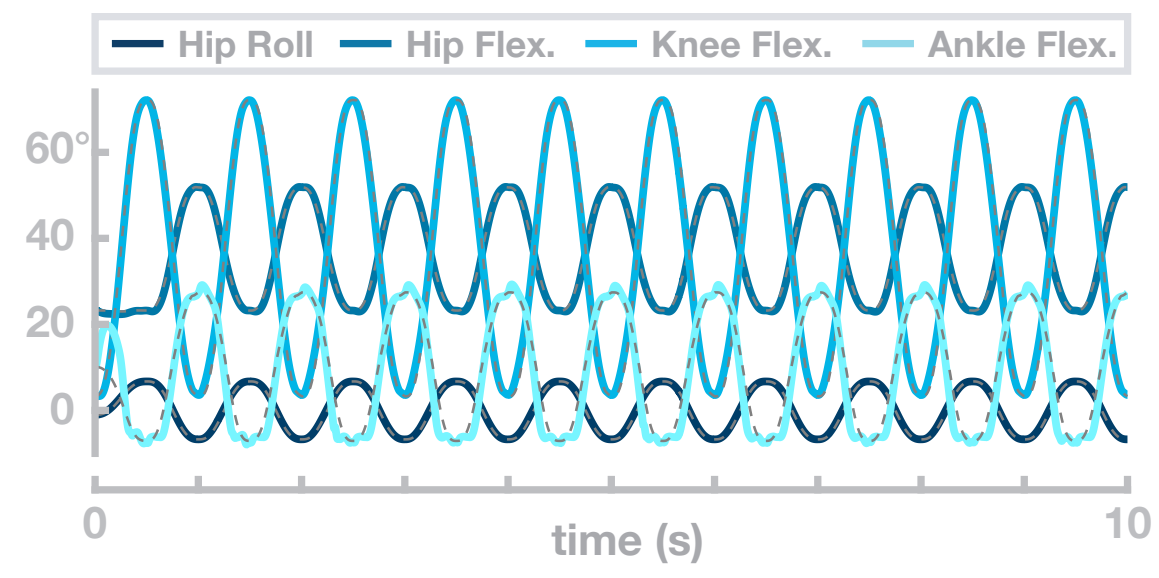

Figure 4: Example movement trajectories of the swing leg with a passively stabilized trunk. The dashed black lines show the planned movement trajectory and the blue lines show the realized joint angle trajectories.

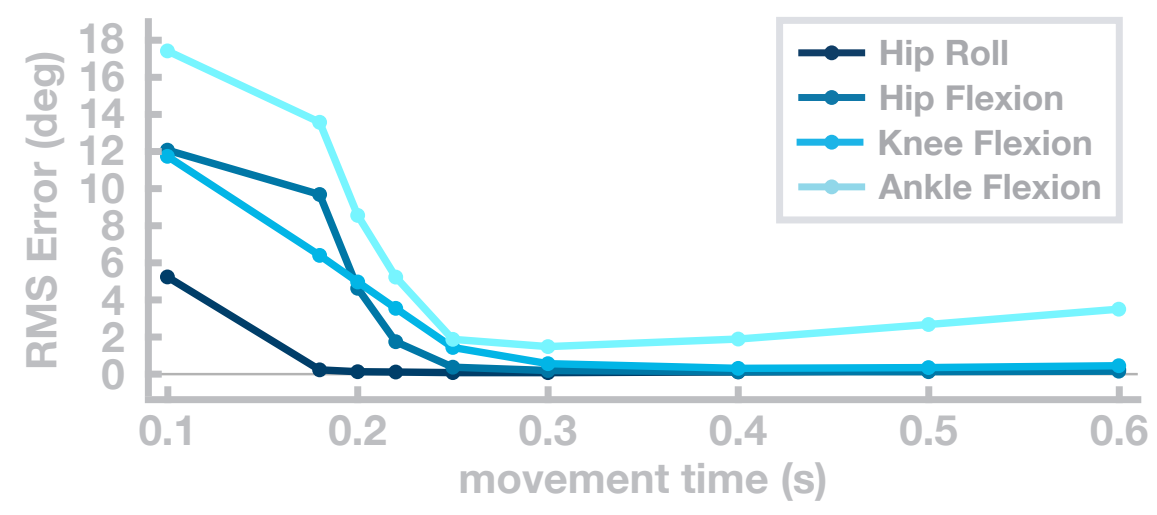

Figure 5: Joint-space error for single movements with different movement times. Each curve shows the root-mean-squared error the respective joint.

the error drops below $1^{\circ}$ for the hip and knee joints. For the ankle joint, the error reaches a minimum of $\approx 2^{\circ}$ at the 0.3 second duration and then increases again.

\subsection{Obstacle Avoidance}

We use an obstacle avoidance task to assess the ability of the model to integrate flexible swing leg movements control of upright balance during walking. We test two different avoidance strategies, (1) lifting the swing leg to step over an obstacle and (2) shifting the swing leg sideways to step around an obstacle. To avoid an obstacle, we adjust the movement plan for the early swing phase by updating the target joint angles $\tilde{\theta}_{i}$ for the early swing phase based on the obstacle position and size. We used a linear mapping

$$
\tilde{\theta}_{i}=\alpha_{i} h_{o}+c_{i},
$$

to determine the target joint angles, where $h_{o}$ is the obstacle extension, i.e. the height for sagittal and the width for medial-lateral avoidance, including a security margin. The joint index $i$ ranges over the ankle, knee and hip flexion degrees of freedom for sagittal and the hip abduction joint for medial-lateral avoidance, and $c_{i}$ is a constant offset. We determined these parameters in an ad-hoc manner based on a few sample movements with hand-fitted values. After mid-swing, foot placement and balance recovery recovery is controlled by the usual balance-control strategy described in Section 2.1.3. We tested obstacle avoidance in both directions on obstacles of different sizes. An example of the model stepping over an obstacle is shown on the right in Figure 1. 

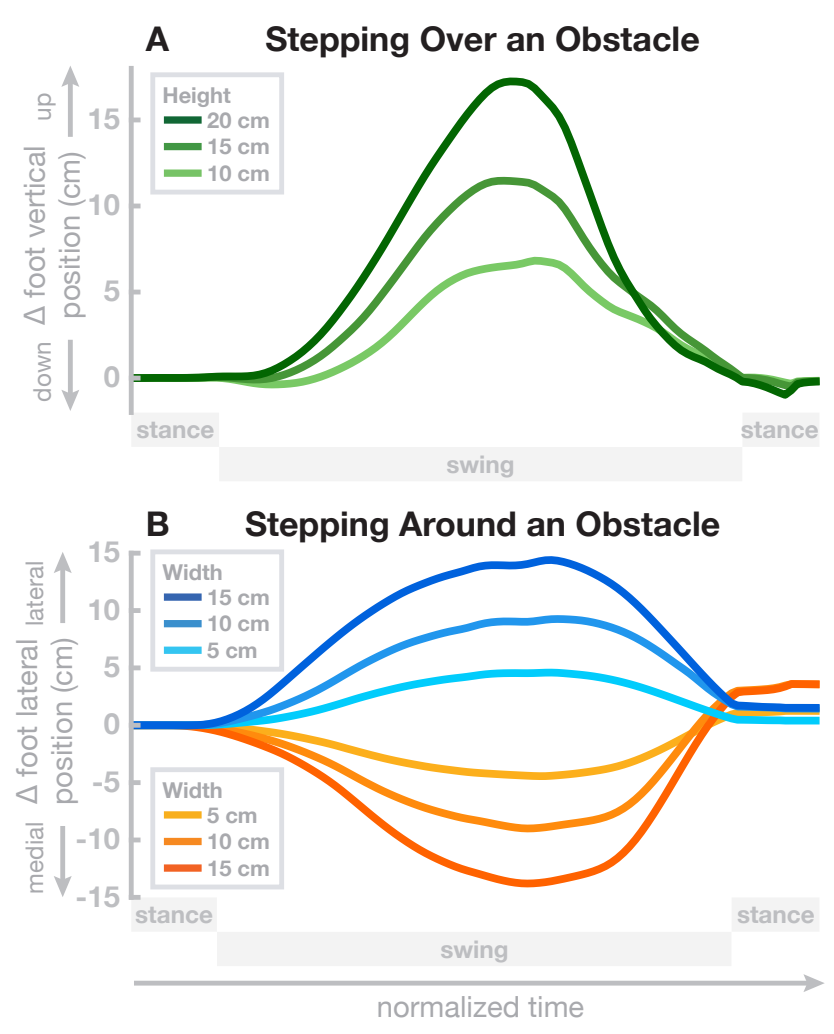

Figure 6: Avoiding obstacle during steady-state walking. Panel A shows the change from the normal trajectory without obstacle in the vertical direction when stepping over obstacles of varying height. Panel B shows the change from the normal trajectory without obstacle in the medial-lateral direction when stepping around obstacles of varying width, in either direction. Both panels show the movement from left heel-strike to push-off of the stance foot.

For sagittal avoidance, we simulated obstacles of $15 \mathrm{~cm}, 20 \mathrm{~cm}$ and $25 \mathrm{~cm}$ height. All three obstacles are successfully avoided and the model returns to the original gait within the subsequent step. Panel A in Figure 6 shows the difference between the balls of the foot relative to the normal movement with no obstacle, in the vertical direction, for these movements. The peaks of these difference plots show that in each movement the balls of the foot are successfully shifted upwards by the obstacle height, plus a safety margin. Note that these vertical positions are differences from the normal foot movement trajectory and the absolute vertical position of the foot is higher, around $\approx 10 \mathrm{~cm}$ at mid-swing.

For medial-lateral avoidance, we simulated obstacles of $5 \mathrm{~cm}$ and $10 \mathrm{~cm}$ width, and both lateral and medial avoidance. Panel B in Figure 6 shows the differences between the balls of the foot relative to the normal movement with no obstacle, in the medial-lateral direction, for these movements. Similar to the sagittal avoidance, the peaks show that the movements are successfully shifted sideways by the desired amount corresponding to the width of the obstacle, plus a safety margin.

\subsection{Direction and Speed Control}

The model has a limited degree of flexibility to walk at different movement speeds and change direction. To change speed, we change the average trunk lean angle by varying the target orientation of the trunk in the spinal reflex module for upright trunk stabilization (see Section 2.2.2 and Song and Geyer (2015)). Generally, increasing the trunk forward lean makes the model walk faster. To explore this relationship, we simulated $40 \mathrm{~s}$ of the model walking with 13 different random values for target orientation of the trunk, drawn from a uniform distribution between $6-8.5^{\circ}$. Figure 7 plots the resulting walking speed of the model in Panel A. Walking speed depends roughly linearly on the trunk, as shown by the linear fit $\left(R^{2}=0.9157\right)$. Panel B in Figure 7 shows how the stepping cadence varies depending on trunk lean for the same walking 


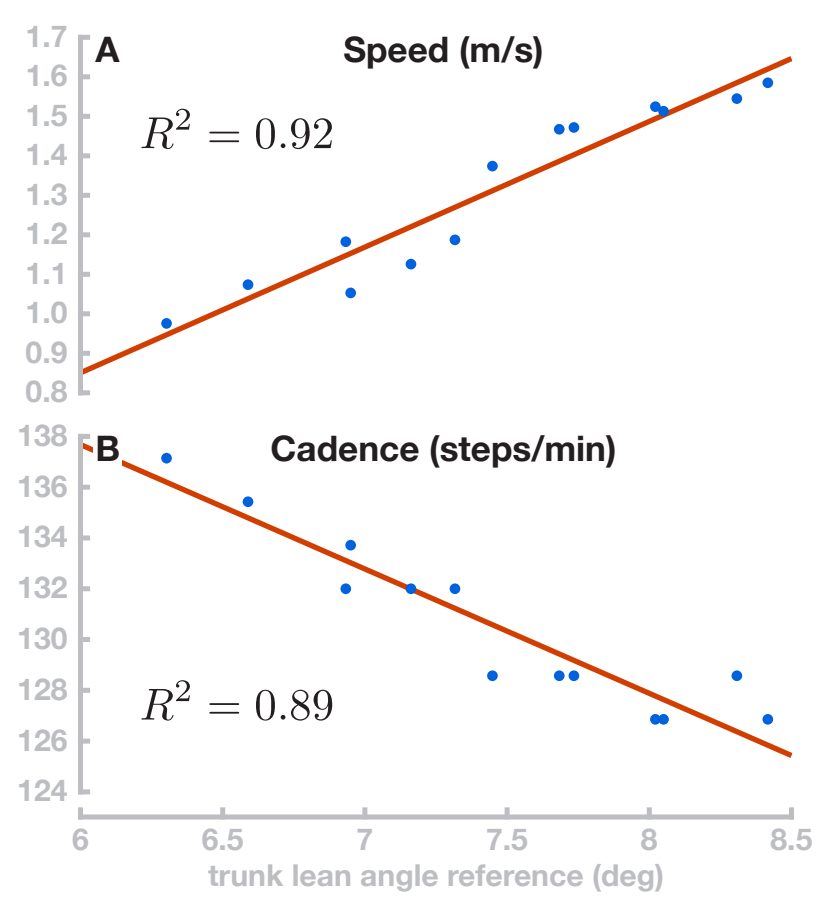

Figure 7: Velocity and cadence control. Panel A shows the relationship between the reference parameter for trunk lean and the resulting movement speed for 13 movements (blue dots) and the linear fit (red line). Panel B shows the effect of the trunk lean change on the walking cadence.

simulations. Interestingly, higher walking speeds are associated with lower cadences. This is opposite to what is observed in humans, where cadence tends to increase with walking speed in normal walking (Nilsson and Thorstensson, 1987). We interpret this as an indicator that the speed variations are not actively controlled, but rather emerge from the interaction of the trunk lean with balance control. Increased trunk lean leads to larger gravitational acceleration and higher speeds, which results in the balance control module increasing the target for the swing leg angle (Equation 1). This generates longer steps and decreases cadence.

Although the model has no rotational degree of freedom at the hip, it is possible to change the direction of movement in a limited fashion. Similar to speed control, we achieve this by exploiting an interaction between movement direction and balance control. Temporarily adding a constant value to the hip roll target angle causes a weak destabilization of the model. This destabilization in turn induces a rotational slight slipping of the stance foot during weight acceptance that results in the body turning. We use this effect in an ad-hoc control law for movement direction that adds this constant offset to the hip roll target angle when the horizontal orientation of the trunk segment lies outside a desired interval around the target direction. We demonstrate this direction control scheme by simulating four walks with different target orientations of $0^{\circ}$, $15^{\circ}, 30^{\circ}$ and $45^{\circ}$, all starting at $0^{\circ}$ and simulated for $100 \mathrm{~s}$. Figure 8 shows the resulting walking patterns. For all four target orientations, the model approximately turns to the target orientation after about $20 \mathrm{~m}$ walking. However, this mode of direction control is not very stable and has clear limitations. The $15 \mathrm{deg}$ and 30 deg movements turn away from the target orientation at about 16 meters of walking even though they reached the target orientation relatively fast after $12 \mathrm{~m}$.

\section{Discussion}

We presented a musculoskeletal model of human locomotion that combines stable walking behaviour with the flexibility to generate voluntary movements with the swing leg according to a kinematic motor plan and to adapt the gait pattern. The model combines biomechanics, muscle physiology, spinal reflex loops and supraspinal neural processes in a physiologically plausible way. The supraspinal layer organizes the behavioural sequence, generates a movement plan on the task level and transforms the movement plan into 
bioRxiv preprint doi: https://doi.org/10.1101/2021.09.26.461864; this version posted September 26, 2021. The copyright holder for this preprint (which was not certified by peer review) is the author/funder, who has granted bioRxiv a license to display the preprint in perpetuity. It is made available under aCC-BY-NC-ND 4.0 International license.
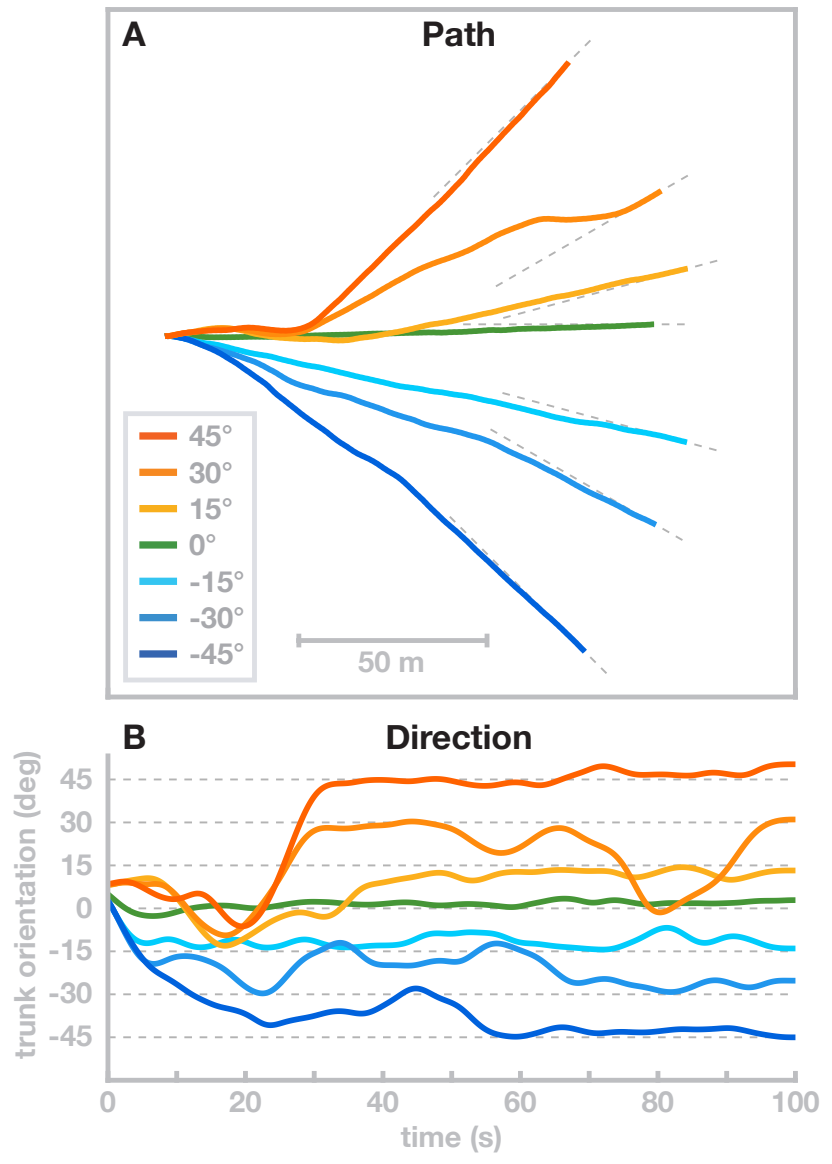

Figure 8: Direction control. Movement direction was adjusted by temporarily adding a constant offset value to the hip roll target angle until the target movement direction was reached, with seven different target directions. Panel A shows the horizontal path of the trunk center. Panel B shows the trunk orientation over time. 
descending motor commands that interface with the spinal cord. The spinal layer combines the descending motor commands for the swing leg with stretch reflex arcs for each muscle by shifting the muscle activation thresholds of the reflexes based on the descending command. Stance leg control is exclusively spinal, consisting of five dedicated reflex modules that each implement a specific function, following (Song and Geyer, 2015). The model generates stable walking patterns and can flexibly move the swing leg according to a kinematic plan to avoid obstacles. It can withstand external perturbations and change walking speed and movement direction to a limited degree.

\subsection{Motor Plans and Voluntary Movements}

The main innovation of the model we presented here is the ability to plan and execute voluntary movements with the swing leg, and to integrate these flexible swing leg movements into a stable gait cycle. Neuroscientists generally differentiate actions into two categories of volitional and reflexive actions (Balleine, 2019). Volitional actions are understood to be goal-directed, model-based and prospective, whereas reflexive actions are habitual, model-free, and retrospective (Dolan and Dayan, 2013). Volitional actions are caused by a desire to reach a certain state in the future, whereas habitual or reflexive actions are caused by a stimulus in the past. While most of this work is at the intersection between neuroscience and psychology and investigates decisions, it intersects the field of motor planning and control.

Walking is largely considered a reflexive movement, although it requires some executive control (Clark, 2015). Decerebrated cats are able to walk without their brains, with only a tonic stimulation of their spinal cords (Whelan, 1996). Models of bipedal locomotion show that reflexes are sufficient to generate stable walking patterns in principle (Günther and Ruder, 2003; Geyer and Herr, 2010; Song and Geyer, 2015). The only high-level modulation required in these models is for balance control. Precise, goal-directed movements, on the other hand, generally require cortical control. When receiving a motor cortex lesion, rodents and primates initially lose the ability to perform goal-directed reaching movements (Whishaw, 2000; Darling et al., 2011). Lesioned animals tend to recover some or large parts of the lost motor function over weeks or months after the lesion, either by local reorganization and neural plasticity (Darling et al., 2011) or by developing compensatory movements (Gharbawie and Whishaw, 2006). Even for goal-directed movement, the brain might not be critical. Kawai et al. (2015) showed that when rats learn a complex sequential lever press movement, they can still execute the learned movement after a lesion to the motor cortex. When receiving the motor cortex lesion before training, however, the rats were unable to perform or learn the lever press movement. Walking can be performed reflexively in steady-state on even or mildly uneven ground. More stringent constraints, such as walking over stepping stones or across a field cluttered with obstacles, require precise movements based on sensory information with a goal of getting the foot precisely onto a stepping stone, or around an obstacle Patla et al. (1991); Chou et al. (2001).

Existing models of walking are mostly reflex-based (Günther and Ruder, 2003; Geyer and Herr, 2010; Song and Geyer, 2015; Ong et al., 2019; Di Russo et al., 2021). The walking movement pattern can be modified to some degree in various models to change speed or step over obstacles, but these modifications are designed for and limited to a specific target behavior. Taga (1998) shows shows that a walking model driven by a neural oscillator can adjust step length by adjusting timing and magnitude of the hip flexor activity, and increase toe clearance by superposing an additional descending motor command to the knee flexor muscles over the rhythmic activity. The model can step over obstacles placed at arbitrary positions by combining modulation of step length and toe clearance, but it lacks the control to move the foot along a specific path. Song and Geyer (2015) show that a model that is almost exclusively controlled by low-level reflexes can be generate stable walking movements in 3d. They achieve balance by modulating the reflex parameters slightly based on high-level information about the body in space. The model is robust in rugged terrain and has a certain degree of adaptability in that it can be made to walk at different speeds and change toe clearance to step over an obstacle. Adaptation is achieved by re-tuning the reflexes that map sensory information to muscle activation to a new cost function using evolutionary optimization Hansen (2006). Effectively, the model learns each behavior individually. Van der Noot et al. (2018) showed that it is possible to generalize between different sets of learned behaviors by interpolating between parameter sets, which generally results in an intermediate behavior. The mechanism can be used to combine the purely reflexive walking generation in this class of model with a degree of central control, that maps a low-dimensional task parameter like walking speed onto a high-dimensional set of reflex parameters that will generate a walking pattern with 
the desired walking speed. But the movements generated by these models are still largely habitual, in that muscle activation is generated reflexively based on the current state of the system, rather than a desired future state and a motor plan for how to get to that state - they still lack the flexibility to plan and execute voluntary movements.

In the work presented here, we developed a model that combines reflexive control of the stance leg with precise, goal-directed movements of the swing leg to generate walking movements that can be flexibly adapted to solve a task. Swing leg movements are planned on task level in the form of minimum jerk trajectories for kinematic task variables. The motor plan is represented as a trajectory that moves the task variable to a desired value in a specified time. For instance, swinging the leg forward is a planned movement of the thigh segment angle in the sagittal plane from a negative value at push-off to the positive value required for a successful heel-strike of the next step. This motor plan is updated during the movement to account for deviations from the planned trajectory of the task variable (see Section 2.1.4), and also to incorporate changes in the goal value required to maintain balance (see Section 2.1.3). To execute this motor plan, an inverse model of the spinal stretch reflex, muscle properties and biomechanics is used to calculate a descending motor command.

Our model has the flexibility to execute any movement plan as a volitional, goal-directed action. It can track random kinematic trajectories with high precision (see Section 3.1) when passively stabilized at the trunk. When moving freely, it can utilize this flexibility to move the leg over and around obstacles during swing. This flexibility is new for a walking model.

The model combines this flexibility with the ability to execute habitual movements, represented by subcortical reflexes that directly map sensory information to muscle activation. Stance leg control is completely reflexive, while swing leg control combines the flexibility of goal-directed movements with the robustness of spinal stretch reflexes. The coordination of these two different types of behavior is organized by state-based switches. The neural mechanisms that implement this ability to smoothly swap be between different types of movement and sequentially combine habitual, reflexive with volitional, goal-directed behavior are thought to be located in the basal ganglia (Lanciego et al., 2012). Impairments to these structures, for instance from cell loss associated with Parkinson's Disease, leads to reduced ability to switch between reflexive and goal-directed behavior, e.g. a reduced ability to voluntarily initiate gait from a standing posture, or the freezing of gait in some people with $\mathrm{PD}$, which predominantly occurs in situations where environmental constraints require a goal-directed, planned modulation of a steady-state gait pattern, such as navigating through a doorway or over an obstacle (Peterson and Horak, 2016; Warabi et al., 2018). A mechanistic understanding of how impairments in neural function lead to specific motor deficits would require a model that encompasses both volitional and habitual movements, the neural mechanisms switching between them, and the integration with the spinal reflexes, muscle physiology and biomechanics that ultimately generate the movement. The model described here represents a first step towards such a mechanistic understanding.

\subsection{Integration of High-level Control and Spinal Reflexes}

For the swing leg control, our model uses a general stretch reflex that increases neural stimulation of the muscle based on the sensory information from muscle spindles and Golgi tendon organs about the length, velocity and force of the muscle (see Equation 16 and Latash, 2008). The descending command $u$ shifts the set-point of this muscle-length feedback loop, and $\dot{u}$ does the same for the velocity feedback. Similar equations have been used in various neuromechanical models of motor control, mostly of the upper limb (Feldman, 1986; Günther and Ruder, 2003; Kistemaker et al., 2007; Buhrmann and Di Paolo, 2014), but also in standing (Reimann and Schöner, 2017) and walking (Günther and Ruder, 2003).

Technically, the formula we use is very similar to the equation used in the equilibrium point hypothesis approach to motor control (Feldman, 1986). This approach postulates that the spinal cord reflex modules simplify the control problem for the high-level areas, so that in order to move a limb to a desired position, the high-level controller only has to specify an equilibrium point corresponding to that position, and the lowlevel spinal reflexes generate the details of the actual movement (Feldman, 1986; Buhrmann and Di Paolo, 2014). Modifications use different patterns of the descending command trajectory, like ramps or N-shapes (Latash and Gottlieb, 1991; Gribble et al., 1998). While more complex, these still adhere to the underlying concept that the structure of the descending command is simple and the spinal cord accounts for most of the complex details of the resulting movement pattern. 
Despite the technical similarity in the stretch reflex, our model differs in the concept behind the equilibrium point hypothesis that the descending commands are simple. We found that considerable complexity is required to successfully generate movements that are both precise and flexible. One source of complexity are the highly non-linear inertial, gravitational and interaction forces that arise during locomotion. In a previous model of balance control in standing that with a similar control approach of shifting thresholds for stretch reflexes, we found that an internal model of the mass distribution and muscle moment arms across the joints and body segments was sufficient to maintain balance (Reimann and Schöner, 2017). Specifically, the model did not include gravitational or velocity dependent interaction forces. Still, the inertial forces alone are sufficiently complex to break the direct correspondence between task-level motor plan and musclelevel control, suggesting that an intermediate step is required to translate the high-level motor plan into descending commands.

In the system presented here, we implemented this intermediate step transforming the high-level motor plan into low-level descending commands with an internal model of the body biomechanics, muscle properties, and the stretch reflex. We do not claim that this is part of our model neurophysiologically plausible. Rather, we see it as a necessary connection between two systems with well-documented neurophysiological functions. There is good evidence that the higher motor areas in the brain plan and monitor movements using a tasklevel representation, e.g. the position or velocity of the hand when reaching to a target (Georgopoulos and Grillner, 1989; Schwartz and Moran, 1999; Churchland et al., 2012; Hodgson and Hogan, 2000). There is similarly good evidence for low-level reflex arcs in the spinal cord, mapping proprioceptive signals directly to $\alpha$-motorneuron activation (Sharbafi and Seyfarth, 2017; Kiehn, 2016). How the high-level movement plan is integrated with the low-level reflexes is currently not well understood (Albert et al., 2020; Ambike et al., 2015; Stollenmaier et al., 2020).

In the present model, we used analytical inversion of the model equations and real-time re-planning for online updating to implement a module that functionally solves this problem of connecting the task-level motor plan with low-level motor areas in the spinal cord. We assume that this functionality is implemented neurally in the actual nervous system, solving the same problem but with a very different internal structure. There is some conceptual overlap with this notion and the equilibrium-point hypothesis, namely that there is a high-level motor control area that plans and generates movement on task level and then hands the details of execution over to more low-level structures. In walking, the present model shows, this transformation is of considerable complexity and needs to be addressed to generate movement patterns that actually walk.

\subsection{Rhythmic Pattern Generation}

In human walking, muscle forces, neural activity and ground reaction forces interact to generate rhythmic movement patterns. Existing approaches to model the dynamics of this combined system fall broadly in two categories, where the rhythmic neural pattern driving the motor system is either generated centrally Taga (1995a), or emerges from the interaction between the body and the ground, fed back into the nervous system via sensory organs Song and Geyer (2015); Geyer and Herr (2010); Ong et al. (2019); Geijtenbeek et al. (2013); Wang et al. (2012). In the first approach, a dedicated neural structure, often called a central pattern generator (CPG), transforms a tonic neural activation into a rhythmic activation pattern between multiple neurons. CPGs are well-documented in insects (Guertin, 2013; Mantziaris et al., 2020). Evidence for CPGs has been found in cats, where a decerebrated cat can still walk when receiving tonic electrical stimulation at certain sites in the spinal cord (Whelan, 1996). Taga (1995a) uses this approach to model human movement. In this model, a bank of neural oscillators drives the activation of the agonist-antagonist muscles spanning the leg joints, with one oscillator per joint. The structure of the neural oscillators broadly follows older models of spinal stepping generators (Miller and Scott, 1977; Kawahara and Mori, 1982), consisting of two neurons, one activating the agonist and one the antagonist muscle of a joint. Such systems have stable oscillation patterns even in the absence of external inputs (Matsuoka, 1985), though in Taga's model both input and output are modulated depending on sensory data and the behavioral state, e.g. stance vs. swing. This model generates stable and robust walking patterns in the sagittal plane and can adapt to uneven terrain and additional loads (Taga, 1995b). Walking speed can be increased by adding tonic input and cadence can be controlled to a limited degree via entrainment by adding a rhythmic input.

In a second category of models, the rhythmic activity does not arise from neural oscillators, but from the interaction between neural control and the environment. In this class of models, muscle force generated by 
reflexes that drive a limb to a desired configuration, e.g. the swing leg forward after pushing off the ground. Different reflexes are turned on and off depending on sensory information, such as the leg switching from swing to stance once contact between the foot and the ground is detected. Organized appropriately, such interaction between reflexes, behavioral switches and environmental contacts generates stable oscillatory patterns. van der Linde (1999) showcase this principle in a biomechanically simple passive walker model with two legs actuated by spring-damper systems, where stable walking patterns emerge passively from the biomechanics, but the stiffness of the damped-spring muscles is increased at certain points in the cycle, based on sensory information, to replace the energy lost to damping back into the system. Günther and Ruder (2003) use more realistic biomechanics with hip, knee and ankle joints that are actuated by Hilltype muscles, with muscle activation determined by generic stretch reflexes. Rhythmic patterns arise from switching between different set-points for the stretch reflexes, triggered by state feedback. Another model by Geyer and Herr (2010) has similar biomechanics, but uses a selection of reflex modules to activate muscles. Each reflex module is designed to fulfill a specific function, activating a small set of muscles based on varied sensory input ranging from muscle length and velocity to forces and joint angles. Song and Geyer (2015) extended this model to 3d, and Van der Noot et al. (2018) combined it with a neural CPG.

The model presented here partially follows the tradition of combining reflexes with behavioral switches to generate rhythmic movement patterns. As some other models, our model shows a limited degree of flexibility in pattern this generation, in that the resulting movement speed can be varied depending on the hip extensor force (Taga, 1995b), the choice of control parameter set (Song and Geyer, 2015; Van der Noot et al., 2018), or in our case the trunk forward lean angle reference (see Section 3.3 above). Taga (1995b) shows that cadence can be modulated as well by entraining the pattern generator to an external signal. The range in which cadence can be modulated in Taga's model is relatively limited, spanning roughly 95-120 steps per minute. More recently, Di Russo et al. (2021) showed that in a reflex model, modulation of a relatively small set of reflex parameters is sufficient to generate a wide range of walking patterns with cadences between $61-118$ steps per minute, speeds between 0.48 and $1.71 \mathrm{~m} / \mathrm{s}$ and step lengths between 0.43 and $0.88 \mathrm{~m}$. While Taga (1995b) varied cadence and speed together, Di Russo et al. (2021) showed some independence, successfully modulating step length at a constant step duration, though failing to modulate step duration at a constant step length.

When humans walk at a certain speed, they will generally use a certain combination of cadence and step length to achieve that speed that is largely invariant across repetitions (Inman et al., 1981). But humans are also capable of walking at different combinations of cadence and step length for a given speed (Nilsson and Thorstensson, 1987), as required e.g. when marching in-step. None of the currently existing models, our own included, is capable of this degree of flexibility. It can be argued that walking with a highly unusual combination of cadence and step length is more of a volitional action than normal walking, and requires motor planning and cortical control, which is largely absent in the existing models of human walking.

\subsection{Scope and Possible Extensions}

We presented a neuromuscular model of human locomotion that combines flexible central control of the swing leg with fast and robust reflexive control of the stance leg. Swing leg movements are realized as goal directed reaching movements and can easily adapt to required task constraints. Stance leg control, on the other hand, is achieved by five spinal reflex modules that (1) generate compliant, spring-like leg behavior, (2) prevent knee overextension, (3) balance the trunk, (4) compensate swing leg interactions and (5) plantarflex the ankle. This purely spinal control of the stance leg has the advantage that the leg can reactively compensate for unpredictable ground reaction forces on a fast time scale, without the need for central integration of different sensory systems, which is time consuming (Peterka, 2002; Carver et al., 2006; van der Kooij and Peterka, 2011). The presented model is limited such that the central controller has no direct access to the stance leg. Adaptations to desired stance leg motion patterns are only possible when reflex gain parameters are changed, requiring the re-optimization of the model parameters. Gaining high-level control over the stance leg could be achieved by superposing the existing reflex modules with additional descending control commands that realize desired gait adaptations while the functional reflex modules remain intact. The superposition of reflex modules and central control has been shown in a model of quite standing (Suzuki and Geyer, 2018) where human sway signatures could be reproduced by combining muscle reflexes and virtual model control. We are currently working on extending the model in this direction to investigate if the superposition of descending 
and reflexive control can be applied to the stance phase of locomotion.

Lateral balance control has been recently found to be governed by three biomechanical control mechanisms: The foot placement mechanism, the push-off-modulation and the ankle roll mechanism (Reimann et al., 2018a). The foot placement mechanism describes an active shift of the lateral foot placement location at footfall after a perturbation (Hof, 2008; Bruijn and van Dieën, 2018). Shifting the footfall position changes the gravitational torque acting on the body through the new stance leg during the following step. This change in gravitational torque compensates for the perturbation. Push-off modulation is a change in the ankle flexion angle of the trailing leg during double stance, starting in late single stance (Kim and Collins, 2015, 2017; Reimann et al., 2018a). An increase in the ankle plantarflexion, for instance, generates a push-off force that shifts the body weight between the two stance legs, in a direction that is largely forward, but also to the side (Reimann et al., 2018b). The lateral component of the body weight shift compensates for lateral perturbations. The ankle roll mechanism is an active ankle inversion/eversion torque at the stance leg in single stance (Hof and Duysens, 2018; Reimann et al., 2018b), activating lateral ankle muscles to pull the foot segment and the rest of the body together. The foot segment rolls on the ground and shifts the CoP compensating for the perturbation. In the presented model, balance control solely relies on the foot-placement mechanism. This demonstrates that both push-off modulation and ankle roll mechanism are functionally not necessary for stable locomotion (Townsend, 1985). However, the two mechanisms are found to play a functional role in human walking increasing lateral stability especially in dedicated phases of the gait cycle. Simulations from simple SLIP models showed that using the ankle mechanism, when available, substantially reduces the amount of foot placement modulation required to maintain balance (Reimann et al., 2017). Adding the push off modulation and ankle roll mechanisms into the current model might improve balance in the model, leading to increased robustness against perturbations, and also lead to a better representation of human behavior by the model.

Human locomotion involves the coordination of multiple muscles spanning the different joints along the legs. Usually there are more muscles than biomechanical degrees of freedom, implying that there are different combinations of muscle forces that will lead to the same torques acting on the joints. Control requires selecting a particular solution out of this abundance of choice (Bernstein, 1967; Latash, 2012; Siciliano and Khatib, 2008). From a biomechanics perspective, specific muscles appear to be particularly appropriate for solving specific motor tasks. For instance, Hof (2001) showed that mono-articular muscles along the leg produce a force on the body center that is directed in the lengthwise direction along the limb, while the force from bi-articular muscles generates a significant transverse component. It is therefore biomechanically reasonable to compensate vertically acting gravitational forces with mono-articular muscles, while using biarticular muscles when horizontal forces are required. E.g., the gastrocnemius muscle is mostly active during push-off, to propel the body mass forward, since this is one of the few situations where the combination of knee flexion and ankle plantarflexion generated by this muscle is functionally useful. Consistent with this general approach, neural evidence for the use of subgroups of muscles for balance control has been found by Sarmadi et al. (2019). Sarmadi et al. (2019) showed that sagittal trunk stabilization during standing is mainly realized with biarticular hip muscles indicating that specific muscle groups might be dedicated to specific motor tasks. The use of muscle subgroup is generally considered as muscle synergies that have been found in walking (Chvatal and Ting, 2013; Ivanenko et al., 2006, 2004) and reaching (d'Avella and Lacquaniti, 2013). But how are these muscle synergies generated by the CNS? Spinal reflex circuits, as implemented in the stance leg in our model and several other models, map a sensory signal to a specific combination of muscles related to a functional motor task, e.g. stabilizing the knee. Even though multiple muscles affect one single joint, fixed reflex circuits define a unique combination of muscles that are recruited together. Such fixed reflex pathways, however, strongly restrict the ability of the limb to perform movements that are not captured by the pre-defined reflex, as discussed above. Specific co-activation patterns between muscles could also be realized by supra-spinal patterns, using specialized neural networks that learn an optimal solution to a specific task or sub-task that is encountered repeatedly with high frequency, such as swinging the leg forward during walking. In the present model we solved the mapping from joint torques to muscle forces in an ad-hoc manner using an iteration approach (see Section 2.1.5). Whether different solutions might provide functional benefits like improved stability or accuracy of voluntary movements requires further study. 


\section{A Parameters}

\section{Author contributions}

Data Curation: RR, HR

Investigation: RR, HR

Resources: JJJ, GS

Validation: RR, HR

Visualization: RR, HR

\section{Model sources}

\section{Acknowledgements}

RR and GS were funded by BMBF grant 01GQ1803. HR and JJJ were funded by the National Science Foundation grant CRCNS 1822568.

Conceptualization: RR, HG, JJJ, GS, HR

Formal Analysis: RR, HR

Funding Acquisition: JJJ, GS, HR

Methodology: RR, HG, GS, RR

Project Administration: JJJ, GS, HR

Software: RRR, HG, RR

Supervision: JJJ, GS, HR

Writing - Original Draft Preparation: RR, HR

Writing - Review \& Editing: RR, HG, JJJ, GS, HR

The model source files are available at https://github.com/hendrikreimann/FlexibleWalker.

\begin{tabular}{|c|c|c|}
\hline Parameter & value & unit \\
\hline$\tau_{\mathrm{A}}$ & 0.01 & $\mathrm{~s}$ \\
$h$ & 0.65 & - \\
$K_{\mathrm{l}}$ & 5 & - \\
$K_{\mathrm{v}}$ & 0.03 & - \\
\hline \hline
\end{tabular}

Table 2: Muscle and Reflex Parameters 
bioRxiv preprint doi: https://doi.org/10.1101/2021.09.26.461864; this version posted September 26, 2021. The copyright holder for this preprint (which was not certified by peer review) is the author/funder, who has granted bioRxiv a license to display the preprint in perpetuity. It is made available under aCC-BY-NC-ND 4.0 International license.

\begin{tabular}{|c|c|c|}
\hline Parameter & value & unit \\
\hline$\phi_{0}$ & 0.44 & $\mathrm{rad}$ \\
$c_{\mathrm{d}, \text { early }}$ & 0.47 & $\mathrm{rad} / \mathrm{m}$ \\
$c_{\mathrm{d}, \text { late }}$ & 0.30 & $\mathrm{rad} / \mathrm{m}$ \\
$c_{\mathrm{v}, \text { early }}$ & 0.17 & $\mathrm{rad} /(\mathrm{m} / \mathrm{s})$ \\
$c_{\mathrm{v}, \text { late }}$ & 0.2 & $\mathrm{rad} /(\mathrm{m} / \mathrm{s})$ \\
\hline$\phi_{0, \text { lat }}$ & 0 & $\mathrm{rad}$ \\
$c_{\mathrm{d} \text {,early,lat }}$ & 0.13 & $\mathrm{rad} / \mathrm{m}$ \\
$c_{\mathrm{d}, \text { late,lat }}$ & 0.30 & $\mathrm{rad} / \mathrm{m}$ \\
$c_{\mathrm{v} \text {,early,lat }}$ & 0.31 & $\mathrm{rad} /(\mathrm{m} / \mathrm{s})$ \\
$c_{\mathrm{v}, \text { late,lat }}$ & 0.34 & $\mathrm{rad} /(\mathrm{m} / \mathrm{s})$ \\
\hline
\end{tabular}

Table 3: Balance Control Parameters

\begin{tabular}{|c|c|c|}
\hline Parameter & value & unit \\
\hline$\alpha_{\text {hip }}$ & -1.5832 & $\mathrm{rad} / \mathrm{m}$ \\
$\alpha_{\text {knee }}$ & -3.6941 & $\mathrm{rad} / \mathrm{m}$ \\
$\alpha_{\text {ankle }}$ & 0 & $\mathrm{rad} / \mathrm{m}$ \\
$\alpha_{\text {hiproll }}$ & -1.2 & $\mathrm{rad} / \mathrm{m}$ \\
$c_{\text {hip }}$ & 2.3899 & $\mathrm{rad}$ \\
$c_{\text {knee }}$ & 2.3085 & $\mathrm{rad}$ \\
$c_{\text {ankle }}$ & 1.25 & $\mathrm{rad}$ \\
$c_{\text {hiproll }}$ & 0 & $\mathrm{rad}$ \\
\hline
\end{tabular}

Table 4: Obstacle Avoidance Parameters

\begin{tabular}{|c|c|c|}
\hline Parameter & value & unit \\
\hline$\mu_{\text {static }}$ & 0.9 & - \\
$\mu_{\text {dynamic }}$ & 0.8 & - \\
Stiffness & 58860 & $\mathrm{~N} / \mathrm{m}$ \\
\hline
\end{tabular}

Table 5: Ground Contact Parameters 


\section{References}

Ackermann, M. and van den Bogert, A. J. (2012). Predictive simulation of gait at low gravity reveals skipping as the preferred locomotion strategy. Journal of Biomechanics, 45(7):1293-1298.

Albert, S. T., Hadjiosif, A. M., Jang, J., Zimnik, A. J., Soteropoulos, D. S., Baker, S. N., Churchland, M. M., Krakauer, J. W., and Shadmehr, R. (2020). Postural control of arm and fingers through integration of movement commands. eLife, 9:e52507. Publisher: eLife Sciences Publications, Ltd.

Allen, J. L. and Ting, L. H. (2016). Why Is Neuromechanical Modeling of Balance and Locomotion So Hard? In Prilutsky, B. I. and Edwards, D. H., editors, Neuromechanical Modeling of Posture and Locomotion, pages 197-223. Springer New York, New York, NY.

Ambike, S., Zatsiorsky, V. M., and Latash, M. L. (2015). Processes underlying unintentional finger-force changes in the absence of visual feedback. Experimental Brain Research, 233(3):711-721.

Aruin, A. S., Forrest, W. R., and Latash, M. L. (1998). Anticipatory postural adjustments in conditions of postural instability. Electroencephalography and Clinical Neurophysiology/Electromyography and Motor Control, 109(4):350-359.

Balleine, B. W. (2019). The Meaning of Behavior: Discriminating Reflex and Volition in the Brain. Neuron, 104(1):47-62.

Barton, S. L., Matthis, J. S., and Fajen, B. R. (2019). Control strategies for rapid, visually guided adjustments of the foot during continuous walking. Experimental Brain Research, 237(7):1673-1690.

Bauby, C. E. and Kuo, A. D. (2000). Active control of lateral balance in human walking. Journal of Biomechanics, 33(11):1433-1440.

Bernstein, N. (1967). The co-ordination and regulation of movements. Pergamon Press, Oxford.

Bouisset, S. and Zattara, M. (1987). Biomechanical study of the programming of anticipatory postural adjustments associated with voluntary movement. Journal of Biomechanics, 20(8):735-742.

Browning, R. C., Baker, E. A., Herron, J. A., and Kram, R. (2006). Effects of obesity and sex on the energetic cost and preferred speed of walking. Journal of Applied Physiology, 100(2):390-398. Publisher: American Physiological Society.

Bruijn, S. M. and van Dieën, J. H. (2018). Control of human gait stability through foot placement. Journal of the Royal Society Interface, 15(143). ISBN: 0000000302.

Buhrmann, T. and Di Paolo, E. A. (2014). Spinal circuits can accommodate interaction torques during multijoint limb movements. Frontiers in Computational Neuroscience, 8.

Carver, S., Kiemel, T., and Jeka, J. J. (2006). Modeling the dynamics of sensory reweighting. Biological cybernetics, 95(2):123-34.

Chou, L.-S., Kaufman, K. R., Brey, R. H., and Draganich, L. F. (2001). Motion of the whole body's center of mass when stepping over obstacles of different heights. Gait \& Posture, 13(1):17-26.

Churchland, M. M., Cunningham, J. P., Kaufman, M. T., Foster, J. D., Nuyujukian, P., Ryu, S. I., and Shenoy, K. V. (2012). Neural population dynamics during reaching. Nature, 487(7405):51-6. Publisher: Nature Publishing Group.

Chvatal, S. A. P. D. and Ting, L. H. P. D. (2013). Common muscle synergies for balance and walking. Frontiers in Computational Neuroscience, 7. Publisher: Frontiers.

Clark, D. J. (2015). Automaticity of walking: functional significance, mechanisms, measurement and rehabilitation strategies. Frontiers in Human Neuroscience, 9. 
Darling, W. G., Pizzimenti, M. A., and Morecraft, R. J. (2011). Functional recovery following motor cortex lesions in non-human primates: experimental implications for human stroke patients. J. Integr. Neurosci., 10:353-384.

d'Avella, A. and Lacquaniti, F. (2013). Control of reaching movements by muscle synergy combinations. Frontiers in Computational Neuroscience, 7.

De Groote, F. and Falisse, A. (2021). Perspective on musculoskeletal modelling and predictive simulations of human movement to assess the neuromechanics of gait. Proceedings of the Royal Society B: Biological Sciences, 288(1946):20202432.

Dean, G. A. (1965). An Analysis of the Energy Expenditure in Level and Grade Walking. Ergonomics, $8(1): 31-47$.

Di Russo, A., Stanev, D., Armand, S., and Ijspeert, A. (2021). Sensory modulation of gait characteristics in human locomotion: A neuromusculoskeletal modeling study. PLOS Computational Biology, 17(5):e1008594.

Dolan, R. and Dayan, P. (2013). Goals and Habits in the Brain. Neuron, 80(2):312-325.

Donelan, J. M., Kram, R., and Kuo, A. D. (2001). Mechanical and metabolic determinants of the preferred step width in human walking. Proceedings of the Royal Society of London. Series B: Biological Sciences, 268(1480):1985-1992.

Donelan, J. M., Shipman, D. W., Kram, R., and Kuo, A. D. (2004). Mechanical and metabolic requirements for active lateral stabilization in human walking. Journal of Biomechanics, 37(6):827-835.

Feldman, A. G. (1986). Once More on the Equilibrium-Point Hypothesis ( $\lambda$ Model) for Motor Control. Journal of Motor Behavior, 18(1):17-54. Publisher: Routledge _eprint: https://doi.org/10.1080/00222895.1986.10735369.

Fukuchi, C. A., Fukuchi, R. K., and Duarte, M. (2018). A public dataset of overground and treadmill walking kinematics and kinetics in healthy individuals. PeerJ, 6:e4640.

Geijtenbeek, T., van de Panne, M., and van der Stappen, A. F. (2013). Flexible muscle-based locomotion for bipedal creatures. ACM Transactions on Graphics, 32(6):1-11.

Georgopoulos, A. P. and Grillner, S. (1989). Visuomotor coordination in reaching and locomotion. Science (New York, N.Y.), 245(4923):1209-1210. ISBN: 0036-8075 (Print) \r0036-8075 (Linking).

Geyer, H. and Herr, H. (2010). A Muscle-reflex model that encodes principles of legged mechanics produces human walking dynamics and muscle activities. IEEE Transactions on Neural Systems and Rehabilitation Engineering, 18(3):263-273. ISBN: 15344320.

Gharbawie, O. A. and Whishaw, I. Q. (2006). Parallel stages of learning and recovery of skilled reaching after motor cortex stroke: "Oppositions" organize normal and compensatory movements. Behavioural Brain Research, 175(2):249-262.

Gribble, P. L., Ostry, D. J., Sanguineti, V., and Laboissière, R. (1998). Are complex control signals required for human arm movement? Journal of Neurophysiology, 79(3):1409-24.

Guertin, P. A. (2013). Central Pattern Generator for Locomotion: Anatomical, Physiological, and Pathophysiological Considerations. Frontiers in Neurology, 3.

Günther, M. and Ruder, H. (2003). Synthesis of two-dimensional human walking: A test of the $\lambda$-model. Biological Cybernetics, 89(2):89-106.

Hansen, N. (2006). The CMA Evolution Strategy: A Comparing Review. In Lozano, J. A., Larrañaga, P., Inza, I., and Bengoetxea, E., editors, Towards a New Evolutionary Computation: Advances in the Estimation of Distribution Algorithms, pages 75-102. Springer Berlin Heidelberg, Berlin, Heidelberg. 
Hodgson, A. and Hogan, N. (2000). A model-independent definition of attractor behavior applicable to interactive tasks. IEEE Transactions on Systems, Man, and Cybernetics, Part C (Applications and Reviews), 30(1):105-118. Conference Name: IEEE Transactions on Systems, Man, and Cybernetics, Part C (Applications and Reviews).

Hof, A. (2001). The force resulting from the action of mono- and biarticular muscles in a limb. Journal of Biomechanics, 34(8):1085-1089.

Hof, A. and Duysens, J. (2018). Responses of human ankle muscles to mediolateral balance perturbations during walking. Human Movement Science, 57:69-82.

Hof, A. L. (2008). The 'extrapolated center of mass' concept suggests a simple control of balance in walking. Human Movement Science, 27(1):112-25.

Hogan, N. (1984). An organizing principle for a class of voluntary movements. Journal of Neuroscience, $4(11): 2745-2754$.

Hunter, L., Hendrix, E., and Dean, J. (2010). The cost of walking downhill: Is the preferred gait energetically optimal? Journal of Biomechanics, 43(10):1910-1915.

Inman, V., Ralston, H., Todd, F., and Lieberman, J. (1981). Human Walking. Williams \& Wilkins.

Ivanenko, Y. P., Poppele, R. E., and Lacquaniti, F. (2004). Five basic muscle activation patterns account for muscle activity during human locomotion. The Journal of Physiology, 556(1):267-282. _eprint: https://physoc.onlinelibrary.wiley.com/doi/pdf/10.1113/jphysiol.2003.057174.

Ivanenko, Y. P., Poppele, R. E., and Lacquaniti, F. (2006). Spinal Cord Maps of Spatiotemporal AlphaMotoneuron Activation in Humans Walking at Different Speeds. Journal of Neurophysiology, 95(2):602618. Publisher: American Physiological Society.

Jankovic, J. (2008). Parkinson's disease: clinical features and diagnosis. Journal of Neurology, Neurosurgery E) Psychiatry, 79(4):368-376.

Kalaska, J. F., Scott, S. H., Cisek, P., and Sergio, L. E. (1997). Cortical control of reaching movements. Current Opinion in Neurobiology, 7(6):849-859.

Kawahara, K. and Mori, S. (1982). A two compartment model of the stepping generator: Analysis of the roles of a stage-setter and a rhythm generator. Biological Cybernetics, 43:225-230.

Kawai, R., Markman, T., Poddar, R., Ko, R., Fantana, A., Dhawale, A., Kampff, A., and Ölveczky, B. (2015). Motor Cortex Is Required for Learning but Not for Executing a Motor Skill. Neuron, 86(3):800-812.

Kiehn, O. (2016). Decoding the organization of spinal circuits that control locomotion. Nature Reviews Neuroscience, 17(4):224-238.

Kim, M. and Collins, S. H. (2015). Once-per-step control of ankle-foot prosthesis push-off work reduces effort associated with balance during walking. Journal of Neuroengineering and Rehabilitation, 12(1):43. ISBN: 1743-0003.

Kim, M. and Collins, S. H. (2017). Once-Per-Step Control of Ankle Push-Off Work Improves Balance in a Three-Dimensional Simulation of Bipedal Walking. IEEE Transactions on Robotics, 33(2):406-418.

Kirtley, C., Whittle, M. W., and Jefferson, R. J. (1985). Influence of walking speed on gait parameters. Journal of Biomedical Engineering, 7(4):282-288.

Kistemaker, D. A., Van Soest, A. K. J., and Bobbert, M. F. (2007). Equilibrium point control cannot be refuted by experimental reconstruction of equilibrium point trajectories. Journal of Neurophysiology, 98(3):1075-82. 
Kung, S. M., Fink, P. W., Legg, S. J., Ali, A., and Shultz, S. P. (2018). What factors determine the preferred gait transition speed in humans? A review of the triggering mechanisms. Human Movement Science, $57: 1-12$.

Lanciego, J. L., Luquin, N., and Obeso, J. A. (2012). Functional Neuroanatomy of the Basal Ganglia. Cold Spring Harbor Perspectives in Medicine, 2(12):a009621-a009621.

Latash, M. L. (2008). Neurophysiological basis of movement. Human Kinetics. Series Title: Neurophysiological Basis of Movement.

Latash, M. L. (2012). The Bliss of Motor Abundance. Experimental Brain Research, 217(1):1-5. arXiv: NIHMS150003 ISBN: 0014-4819.

Latash, M. L. and Gottlieb, G. L. (1991). Reconstruction of shifting elbow joint compliant characteristics during fast and slow movements. Neuroscience, 43(2):697-712.

Lawson, C. and Hanson, R. (1995). Solving Least Squares Problems. Classics in Applied Mathematics. Society for Industrial and Applied Mathematics.

Levine, D., Richards, J., and Whittle, M. (2012). Whittle's Gait Analysis - E-Book. Elsevier Health Sciences.

Mantziaris, C., Bockemühl, T., and Büschges, A. (2020). Central pattern generating networks in insect locomotion. Developmental Neurobiology, 80(1-2):16-30.

Matsuoka, K. (1985). Sustained oscillations generated by mutually inhibiting neurons with adaptation. Biological Cybernetics, 52:367-376.

Matthis, J. S. and Fajen, B. R. (2014). Visual control of foot placement when walking over complex terrain. Journal of Experimental Psychology: Human Perception and Performance, 40(1):106-115.

Miller, S. and Scott, P. (1977). The spinal locomotor generator. Experimental Brain Research, 30-30(2-3).

Moissenet, F., Leboeuf, F., and Armand, S. (2019). Lower limb sagittal gait kinematics can be predicted based on walking speed, gender, age and BMI. Scientific Reports, 9(1):9510. Bandiera_abtest: a Cc_license_type: cc_by Cg_type: Nature Research Journals Number: 1 Primary_atype: Research Publisher: Nature Publishing Group Subject_term: Biomedical engineering;Computational models Subject_term_id: biomedical-engineering;computational-models.

Mowbray, R., Gottwald, J. M., Zhao, M., Atkinson, A. P., and Cowie, D. (2019). The development of visually guided stepping. Experimental Brain Research, 237(11):2875-2883.

Murray, R. M., Li, Z., and Sastry, S. S. (1994). A Mathematical Introduction to Robotic Manipulation. CRC Press INC.

Mutha, P. K. (2017). Reflex Circuits and Their Modulation in Motor Control: A Historical Perspective and Current View. Journal of the Indian Institute of Science, 97(4):555-565.

Nielsen, J. B. (2003). How we Walk: Central Control of Muscle Activity during Human Walking. The Neuroscientist, 9(3):195-204.

Nilsson, J. and Thorstensson, A. (1987). Adaptability in frequency and amplitude of leg movements during human locomotion at different speeds. Acta Physiologica Scandinavica, 129(1):107-114.

Ong, C. F., Geijtenbeek, T., Hicks, J. L., and Delp, S. L. (2019). Predicting gait adaptations due to ankle plantarflexor muscle weakness and contracture using physics-based musculoskeletal simulations. PLOS Computational Biology, 15(10):e1006993.

Osoba, M. Y., Rao, A. K., Agrawal, S. K., and Lalwani, A. K. (2019). Balance and gait in the elderly: A contemporary review. Laryngoscope investigative otolaryngology, 4(1):143-153. Publisher: Wiley Online Library. 
Patla, A. E., Prentice, S. D., Robinson, C., and Neufeld, J. (1991). Visual control of locomotion: Strategies for changing direction and for going over obstacles. Journal of Experimental Psychology: Human Perception and Performance, 17(3):603-634.

Peterka, R. J. (2002). Sensorimotor integration in human postural control. Journal of Neurophysiology, 88(3):1097-118. ISBN: 0022-3077 (Print) \n0022-3077 (Linking).

Peterson, D. S. and Horak, F. B. (2016). Neural Control of Walking in People with Parkinsonism. Physiology, 31(2):95-107.

Pijnappels, M., Reeves, N. D., Maganaris, C. N., and van Dieën, J. H. (2008). Tripping without falling; lower limb strength, a limitation for balance recovery and a target for training in the elderly. Journal of Electromyography and Kinesiology, 18(2):188-196.

Prentice, S. D., Hasler, E. N., Groves, J. J., and Frank, J. S. (2004). Locomotor adaptations for changes in the slope of the walking surface. Gait \& Posture, 20(3):255-265.

Ralston, H. J. (1958). Energy-speed relation and optimal speed during level walking. Internationale Zeitschrift für angewandte Physiologie einschließlich Arbeitsphysiologie, 17(4):277-283.

Reimann, H., Fettrow, T., and Jeka, J. J. (2018a). Strategies for the Control of Balance During Locomotion. Kinesiology Review, 7(1):18-25.

Reimann, H., Fettrow, T., Thompson, E. D., Agada, P., McFadyen, B. J., and Jeka, J. J. (2017). Complementary mechanisms for upright balance during walking. PLoS ONE, pages 1-16.

Reimann, H., Fettrow, T., Thompson, E. D., and Jeka, J. J. (2018b). Neural Control of Balance During Walking. Frontiers in Physiology, 9(September):1271.

Reimann, H., Ramadan, R., Fettrow, T., Hafer, J. F., Geyer, H., and Jeka, J. J. (2020). Interactions Between Different Age-Related Factors Affecting Balance Control in Walking. Frontiers in Sports and Active Living, 2:94.

Reimann, H. and Schöner, G. (2017). A multi-joint model of quiet, upright stance accounts for the "uncontrolled manifold" structure of joint variance. Biological Cybernetics, 111(5-6):389-403. Publisher: Springer Berlin Heidelberg.

Reynolds, R. F. and Day, B. L. (2005a). Rapid visuo-motor processes drive the leg regardless of balance constraints. Current Biology, 15(2):R48-R49.

Reynolds, R. F. and Day, B. L. (2005b). Visual guidance of the human foot during a step: Visually guided stepping. The Journal of Physiology, 569(2):677-684.

Sabes, P. (2000). The planning and control of reaching movements. Current Opinion in Neurobiology, 10(6):740-746.

Sarmadi, A., Schumacher, C., Seyfarth, A., and Sharbafi, M. A. (2019). Concerted Control of Stance and Balance Locomotor Subfunctions - Leg Force as a Conductor. IEEE Transactions on Medical Robotics and Bionics, 1(1):49-57. Conference Name: IEEE Transactions on Medical Robotics and Bionics.

Schwartz, A. B. and Moran, D. W. (1999). Motor cortical activity during drawing movements: population representation during lemniscate tracing. Journal of Neurophysiology, 82(5):2705-18.

Scott, S. H. (2004). Optimal feedback control and the neural basis of volitional motor control. Nature reviews. Neuroscience, 5(7):532-46.

Sharbafi, M. A. and Seyfarth, A. (2017). Bioinspired Legged Locomotion: Models, Concepts, Control and Applications. Butterworth-Heinemann. Google-Books-ID: 3gVQCwAAQBAJ.

Siciliano, B. and Khatib, O. (2008). Springer Handbook of Robotics. Springer. Series Title: Gale virtual reference library. 
Smid, K. and den Otter, A. (2013). Why you need to look where you step for precise foot placement: The effects of gaze eccentricity on stepping errors. Gait \& Posture, 38(2):242-246.

Song, S. and Geyer, H. (2015). A neural circuitry that emphasizes spinal feedback generates diverse behaviours of human locomotion. Journal of Physiology, 593:3493-3511. ISBN: 0022-3751.

Steele, K. M., van der Krogt, M. M., Schwartz, M. H., and Delp, S. L. (2012). How much muscle strength is required to walk in a crouch gait? Journal of Biomechanics, 45(15):2564-2569.

Stein, R. B. (1991). Reflex Modulation During Locomotion: Functional Significance§§The original work reviewed here was done with Dr. J. Yang, Dr. M. Edamura and Dr. C. Capaday. Support for the research was provided by the Medical Research Council of Canada. Much of this material was presented in Tokyo at a conference on the Neurobiology of Human Locomotion, the proceedings of which are being published by Japanese Scientific Societies Press. In Patla, A. E., editor, Advances in Psychology, volume 78 of Adaptability of Human Gait, pages 21-36. North-Holland.

Stollenmaier, K., Ilg, W., and Haeufle, D. F. B. (2020). Predicting Perturbed Human Arm Movements in a Neuro-Musculoskeletal Model to Investigate the Muscular Force Response. Frontiers in Bioengineering and Biotechnology, 8. Publisher: Frontiers.

Summerside, E. M., Kram, R., and Ahmed, A. A. (2018). Contributions of metabolic and temporal costs to human gait selection. Journal of The Royal Society Interface, 15(143):20180197. Publisher: Royal Society.

Sutherland, D. H. and Davids, J. R. (1993). Common gait abnormalities of the knee in cerebral palsy. Clin Orthop Relat Res., 288:139-47.

Suzuki, Y. and Geyer, H. (2018). A simple bipedal model for studying control of gait termination. Bioinspiration $\&$ Biomimetics, 13(3):036005.

Taga, G. (1995a). A model of the neuro-musculo-skeletal system for human locomotion. I. Emergence of basic gait. Biological Cybernetics, 111:97-111.

Taga, G. (1995b). A model of the neuro-musculo-skeletal system for human locomotion. II. Real-time adaptability under various constraints. Biological Cybernetics, 73:113-121.

Taga, G. (1998). A model of the neuro-musculo-skeletal system for anticipatory adjustment of human locomotion during obstacle avoidance. Biological cybernetics, 78(1):9-17. ISBN: 0340-1200.

Townsend, M. A. (1985). Biped gait stabilization via foot placement. Journal of Biomechanics, 18(1):21-38. ISBN: 0021-9290.

van der Kooij, H. and Peterka, R. J. (2011). Non-linear stimulus-response behavior of the human stance control system is predicted by optimization of a system with sensory and motor noise. Journal of Computational Neuroscience, 30(3):759-778.

van der Linde, R. Q. (1999). Passive bipedal walking with phasic muscle contraction. Biological Cybernetics, $81(3): 227-237$.

Van der Noot, N., Ijspeert, A. J., and Ronsse, R. (2018). Bio-inspired controller achieving forward speed modulation with a 3D bipedal walker. International Journal of Robotics Research, 37(1):168-196.

Voloshina, A. S., Kuo, A. D., Daley, M. A., and Ferris, D. P. (2013). Biomechanics and energetics of walking on uneven terrain. Journal of Experimental Biology, 216(21):3963-3970.

Wang, J. M., Hamner, S. R., Delp, S. L., and Koltun, V. (2012). Optimizing locomotion controllers using biologically-based actuators and objectives. ACM Transactions on Graphics, 31(4):25:1-25:11.

Wang, Y. and Srinivasan, M. (2014). Stepping in the direction of the fall: the next foot placement can be predicted from current upper body state in steady-state walking. Biology Letters, 10:20140405. 
Warabi, T., Furuyama, H., Sugai, E., Kato, M., and Yanagisawa, N. (2018). Gait bradykinesia in Parkinson's disease: a change in the motor program which controls the synergy of gait. Experimental Brain Research, 236(1):43-57.

Whelan, P. (1996). Control of Locomotion in the Decerebrate Cat. Progress in Neurobiology, 49(5):481-515.

Whishaw, I. Q. (2000). Loss of the innate cortical engram for action patterns used in skilled reaching and the development of behavioral compensation following motor cortex lesions in the rat. Neuropharmacology, 39(5):788-805.

Woollacott, M., Bonnet, M., and Yabe, K. (1984). Preparatory process for anticipatory postural adjustments: Modulation of leg muscles reflex pathways during preparation for arm movements in standing man. Experimental Brain Research, 55(2).

Yin, K., Loken, K., and Panne, M. V. D. (2007). SIMBICON: simple biped locomotion control. In ACM SIGGRAPH 2007 papers, volume ab, page 105, San Diego, California.

Zehr, E. P. and Stein, R. B. (1999). What functions do reflexes serve during human locomotion? Progress in Neurobiology, 58(2):185-205.

Zhang, Y., Smeets, J. B. J., Brenner, E., Verschueren, S., and Duysens, J. (2020). Fast responses to steppingtarget displacements when walking. The Journal of Physiology, 598(10):1987-2000. 